\title{
Detection and blind channel estimation for UAV-aided wireless sensor networks in smart cities under mobile jamming attack
}

This paper was downloaded from TechRxiv (https://www.techrxiv.org).

\section{LICENSE}

CC BY 4.0

SUBMISSION DATE / POSTED DATE

$27-10-2021 / 01-11-2021$

\section{CITATION}

Darsena, Donatella; Gelli, Giacinto; ludice, Ivan; Verde, Francesco (2021): Detection and blind channel estimation for UAV-aided wireless sensor networks in smart cities under mobile jamming attack. TechRxiv. Preprint. https://doi.org/10.36227/techrxiv.16884496.v1

$\mathrm{DOI}$ 


\title{
Detection and blind channel estimation for UAV-aided wireless sensor networks in smart cities under mobile jamming attack
}

\author{
Donatella Darsena, , Giacinto Gelli, , Ivan Iudice, \\ and Francesco Verde,
}

\begin{abstract}
Unmanned aerial vehicles (UAVs) can be integrated into wireless sensor networks (WSNs) for smart city applications in several ways. Among them, a UAV can be employed as a relay in a "store-carry and forward" fashion by uploading data from ground sensors and metering devices and, then, downloading it to a central unit. However, both the uploading and downloading phases can be prone to potential threats and attacks. As a legacy from traditional wireless networks, the jamming attack is still one of the major and serious threats to UAV-aided communications, especially when also the jammer is mobile, e.g., it is mounted on a UAV or inside a terrestrial vehicle. In this paper, we investigate anti-jamming communications for UAV-aided WSNs operating over doubly-selective channels in the downloading phase. In such a scenario, the signals transmitted by the UAV and the malicious mobile jammer undergo both time dispersion due to multipath propagation effects and frequency dispersion caused by their mobility. To suppress high-power jamming signals, we propose a blind physical-layer technique that jointly detects the UAV and jammer symbols through serial disturbance cancellation based on symbol-level post-sorting of the detector output. Amplitudes, phases, time delays, and Doppler shifts - required to implement the proposed detection strategy - are blindly estimated from data through the use of algorithms that exploit the almostcyclostationarity properties of the received signal and the detailed structure of multicarrier modulation format. Simulation results corroborate the anti-jamming capabilities of the proposed method, for different mobility scenarios of the jammer.

Index Terms-Almost-cyclostationarity, doubly-selective channels, interference cancellation, mobile jamming, unmanned aerial vehicle (UAV), smart cities, wireless sensor networks.
\end{abstract}

\section{INTRODUCTION}

Wireless sensor networks (WSNs) are expected to play a fundamental role in realizing the vision of future smart cities [1]-[4], which represent one of the major application of Internet-of-Things (IoT). In a smart city, a huge number of sensors and metering devces of various nature are placed at a large variety of locations, such as buildings, parking areas, traffic lights, railway/metro/bus stations, monitoring hubs along roads, and so on, whose task is to gather data to be transmitted towards a powerful central unit (CU) located somewhere in the city. Processing of the collected data at the $\mathrm{CU}$ enables provision of many innovative services within health, transportation, sustainability, economy, agriculture, law enforcement, community, and other areas affecting the overall wellbeing of the residents and businesses. Recently, it has been pointed out the crucial role of smart cities in reducing the COVID-19 risk [5].
Despite the promising service features, the problem of efficient and reliable transmission to a central unit of the data independently collected by sensors and meters remains a challenging issue in IoT applications [6]. One possible option is represented by the LoRa/LoRaWAN technology stack [7], due to its long signal range and minimal power requirements. However, it is common belief that, due to their limited capacity, these technologies could be unable to support the high data-rates required by future smart city applications. In this respect, the employment of unmanned aerial vehicles $(U A V s)$ as aerial "store-carry and forward" relay nodes for distributed ground sensors and meters is expected to bring significant benefits to future WSNs, such as higher data transmissions, larger service coverage, and reduced energy consumption [8], [9]. According to this paradigm, UAVs can move between a sensor/meter and the $\mathrm{CU}$, by gathering data from the sources (uploading phase), and, then, transmitting them to the destination (downloading phase).

Unfortunately, while the enabling technologies for UAVaided WSNs gradually mature, all kinds of potential threats or attacks will also rise and endanger smart city applications [10]. These adversarial behaviors are due to malicious users, such as criminals, terrorists, and business spies, and are driven by various motivations, such as committing crimes, jeopardizing public safety, invading secret databases, and so on.

\section{A. Jamming attacks and related countermeasures}

One serious threat to UAV-aided WSNs is the jamming attack. Radio jamming can be divided into link-layer and physical-layer jamming. The aim of link-layer jamming is to decrease the packet send/delivery ratio [11], [12]. However, these attacks are based on the assumption that the jammer is aware of the link-layer protocol of the legitimate user, which could be an excessively pessimistic assumption. On the other hand, physical-layer jamming methods are very effective in UAV-aided WSNs, since a jammer might emit high-power electromagnetic signals to make the legitimate signals unrecognizable for the UAV in the uploading phase and/or for the $\mathrm{CU}$ in the downloading one. Furthermore, due to the rapid development of software-defined radio techniques, a smart jammer is able to modify the attack pattern according to the transmission features of the targeted communication links. 
Because of the ease of implementation and disruptive impact, physical-layer jamming attacks in UAV-aided WSNs have received a lot of attention and the countermeasures have been extensively studied. Some works rely on strategies implemented at the application layer to protect networks from external attacks. A viable indicator to verify the existence of jamming attacks in WSNs is represented by the packet loss ratio [13]. An intelligent security scheme has been proposed in [14], which uses random neural networks to detect malicious activities in the network and verify the authenticity and legitimacy of the network traffic. A lightweight authentication scheme has been proposed [15] in a cloud-based IoT infrastructure to ensure the security of a collected data from a sensor at a remote location by means of a one-way cryptographic hash function authentication process. In [16], a one-way cryptographic hash function with addition of bitwise exclusiveOR (XOR) operation to verify the legitimacy of participating devices in the network has been developed. However, applying such application-layer countermeasures to UAV-aided WSNs is very difficult, due to their limited resources and dynamic nature. Moreover, they are mainly targeted at identifying the presence of a jamming attack and, thus, are not able to avoid and/or combat it. Furthermore, most of them are specific to a given environment, system, or software, and exhibit a complex authentication process, which generates network overhead. Therefore, innovative anti-jamming strategies are needed at the physical layer to counteract jamming threats.

Anti-jamming physical-layer methods can be categorized as proactive or reactive ones. Proactive strategies either hide signals so that the jammer cannot detect them (like, e.g., in direct sequence spread spectrum) or avoid the jamming attack by periodically changing channels with an interval of fixed length, without checking for jammers [12] (like, e.g., in frequency-hopping spread spectrum). On the other hand, reactive strategies suppress the jamming signal by signal processing techniques or combat the jamming attack directly, e.g., by increasing the transmission power.

Proactive approaches are not particularly suited to UAVaided WSNs, due to their scarce adaptability to dynamic spectrum state and jamming patterns, as well as for their low spectral efficiency [17]. As to reactive approaches for conventional wireless networks, they can be classified into two broad families. Techniques belonging to the first family aim at optimizing the transmission power for the legitimate user and/or at developing efficient channel-selection strategies after detection of channel jamming. In [18], the power-control problem for the legitimate user was studied from a Stackelberg game perspective. By utilizing the spectrum waterfall representation, an anti-jamming scheme based on deep reinforcement learning (RL) method was proposed in [19] to facilitate the channel-selection process. A multi-armed bandit framework was formulated in [20] to obtain efficient channel-selection strategies. In [21], a multi-domain anti-jamming scheme that tackles both power control and channel selection was proposed for heterogeneous wireless networks.

Reputation-based anti-jamming methods belong to the sec- ond family. In [22], a reputation updating process was developed for large-scale wireless networks in order to face with a wide range of attacks. The problem of establishing the defender's reputation in anomaly detection against insider attacks was addressed in [23]. However, due to different network characteristics, such methods are not directly applicable to UAV-aided WSNs.

Specific countermeasures against jamming attacks in UAVaided communications have been proposed in many recent papers. In [24], power control for anti-jamming problem in a pure UAV communication network was investigated from a game theoretic perspective. Multi-parameter programming and RL was used in [25] to protect against multiple jammers the communication between a UAV swarm and a base station. A UAV-aided cellular framework was studied in [26], in which a UAV uses RL to choose the relay policy for a mobile user whose serving base station is attacked by a jammer. In [27], anti-jamming strategies based on RL were investigated to improve UAV-enabled communications against smart jamming attacks. By taking advantage of the flexible mobility patterns of UAVs, anti-jamming trajectories were designed in [28][30] to prevent ground jammers from degrading the legitimate transmission in UAV-enabled communication systems.

Many of the above works assume a non-dispersive channel model for ground-to-air (G2A) and air-to-ground (A2G) links. However, such an ideal assumption is inaccurate in smart city applications, where UAVs are expected to be deployed in urban areas with high and dense buildings or obstacles. In these scenarios, since UAVs are highly mobile in nature, the G2A/A2G channels are better modeled as doubly-selective [31] channels, with multipath effects causing time dispersion and Doppler shifts giving rise to frequency dispersion of the information-bearing signals. Additionally, the jammer can be mobile in its turn, e.g., jamming radios can be piggybacked on a UAV or installed inside terrestrial vehicles. Mobility allows the jammer to rapidly move around the city and complicates localization of the jamming source compared to the case of a fixed jammer.

Henceforth, mobile jamming attacks specifically targeted at UAV-aided WSNs remain a critical issue, and effective anti-jamming reactive methods operating over doubly-selective channels are an open research issue.

\section{B. Anti-jamming communications in smart city applications}

In this paper, we focus on anti-jamming communications by explicitly taking into account the dispersive nature of the wireless channel in smart city applications. We assume that the mobile jammer itself is smart, in the sense that it is able to replicate the same modulation format of the legitimate transmission [12]. Since multicarrier modulation is currently adopted in many wireless standards, we focus on the orthogonal frequency-division multiplexing (OFDM) transmission format. In this case, besides the adverse effects of the jamming signal, due to the UAV speed and fast fading channel conditions, the performance of the multicarrier communication system is also deteriorated by Doppler frequency shifts. 
TABLE I

LIST OF THE MAIN NOTATIONS USED THROUGHOUT THE PAPER.

\begin{tabular}{|c|c|}
\hline Notation & Meaning \\
\hline$x$ & scalar value \\
\hline A & matrix - bold, capital letter \\
\hline a & vector - bold, small letter \\
\hline$x^{*}$ & conjugate of $x$ \\
\hline $\mathbf{A}^{\mathrm{T}}$ & transpose of $\mathbf{A}$ \\
\hline $\mathbf{A}^{\mathrm{H}}$ & Hermitian (conjugate transpose) of $\mathbf{A}$ \\
\hline $\mathbf{A}^{-1}$ & inverse of $\mathbf{A}$ \\
\hline $\mathbb{C}$ & fields of complex numbers \\
\hline $\mathbb{R}$ & fields of real numbers \\
\hline $\mathbb{Z}$ & fields of integer numbers \\
\hline $\mathbb{C}^{n}$ & vector-space of all $n$-column vectors with complex coordinates \\
\hline $\mathbb{R}^{n}$ & vector-space of all $n$-column vectors with real coordinates \\
\hline $\mathbb{C}^{n \times m}$ & vector-space of all the $n \times m$ matrices with complex elements \\
\hline $\mathbb{R}^{n \times m}$ & vector-space of all the $n \times m$ matrices with real elements \\
\hline$\delta_{n}$ & Kronecker delta \\
\hline$j \triangleq \sqrt{-1}$ & denotes the imaginary unit \\
\hline $\max (x, y)$ & maximum between $x \in \mathbb{R}$ and $y \in \mathbb{R}$ \\
\hline$|x|$ & rounds $x \in \mathbb{R}$ to the nearest integer less than or equal to $x$ \\
\hline $\bmod 1$ & denotes modulo 1 operation with values in $[-1 / 2,1 / 2)$ \\
\hline $\mathcal{O}$ & Landau symbol \\
\hline * $\quad$ 2 & linear convolution operator \\
\hline $\mathcal{A}^{n}=\mathcal{A} \times \cdots \times \mathcal{A}$ & $n$th Cartesian power of the set $\mathcal{A}$ \\
\hline & $n$-column zero vector \\
\hline $\mathbf{O}_{n \times m}$ & $n \times m$ zero matrix \\
\hline $\mathbf{I}_{n}$ & $n \times n$ identity matrix \\
\hline $\mathbf{W}_{n}$ & unitary symmetric $n$-point inverse discrete Fourier transform (IDFT) matrix \\
\hline $\mathbf{W}_{n}^{-1}=\mathbf{W}_{n}^{\mathrm{H}}$ & $n$-point discrete Fourier transform (DFT) matrix \\
\hline$\{\mathbf{a}\}_{\ell}$ & $\ell$ th entry of $\mathbf{a} \in \mathbb{C}^{n}$ \\
\hline$\{\mathbf{A}\}_{\ell, \ell}$ & $\ell$ th diagonal entry \\
\hline$\lambda_{\max }(\mathbf{A})$ & largest eigenvalue of $\mathbf{A} \in \mathbb{C}^{n \times n}$ \\
\hline $\operatorname{trace}(\mathbf{A})$ & trace of $\mathbf{A} \in \mathbb{C}^{n \times n}$ \\
\hline $\operatorname{rank}(\mathbf{A})$ & rank of $\mathbf{A} \in \mathbb{C}^{n \times m}$ \\
\hline$\|\mathbf{A}\| \triangleq\left[\operatorname{tr}\left(\mathbf{A} \mathbf{A}^{\mathrm{H}}\right)\right]^{1 / 2}$ & (induced) Frobenius norm of $\mathbf{A} \in \mathbb{C}^{n \times m}$ [42] \\
\hline $\mathbf{A}=\operatorname{diag}\left(a_{0}, a_{1}, \ldots, a_{n-1}\right)$ & $n \times n$ diagonal matrix \\
\hline $\operatorname{vec}(\mathbf{A})$ & concatenation of the columns of $\mathbf{A} \in \mathbb{C}^{n \times m}$ \\
\hline$\langle\cdot\rangle$ & infinite-time temporal averaging \\
\hline $\begin{array}{l}\mathbb{E}[\cdot] \\
x(t)\end{array}$ & $\begin{array}{l}\text { ensemble averaging } \\
\text { continuous-time signals }\end{array}$ \\
\hline$x[n]$ & discrete-time signals \\
\hline
\end{tabular}

The proposed anti-jamming solution relies on a known information-theoretic result [32], according to which, when the signal-to-jammer ratio (SJR) is sufficiently low, the optimal detection strategy of the legitimate transmission (in the minimum-bit-error-rate sense) consists of first estimating the jammer signal and, then, subtracting it from the received data. What is challenging for the problem at hand is that, in order to implement such a jamming-resistant receiver, all the amplitudes, phases, time delays, and Doppler shifts of both the legitimate and jamming channels have to be accurately estimated at the receiver.

Traditional training-based approaches for parameter estimation (see, e.g., [33]) could not work well in UAV-aided WSNs under a jamming attack, since communication resources may be significantly wasted in doubly-selective channels and, most importantly, the jamming attack during the training phase might induce an unacceptable estimation error. Motivated by these facts, to estimate all the parameters of interest of the legitimate and jamming signals, we propose a blind estimation algorithm that relies on the almost-cyclostationarity (ACS) properties [34] of the received signal.
The proposed algorithm capitalizes on two distinct features of UAV-aided WSNs for smart city applications: (i) the availability of a large amount of data, as a consequence of the widespread dissemination of sensors and meters; (ii) the relaxed latency constraints [35], due to the fact that the network scope is not to react to an instantaneous event but rather to monitor the history of sensed data, based on which it is possible to provide new services or to optimize resource consumption. To the best of our knowledge, this is the first paper that investigates the application of ACS properties to achieve anti-jamming communications in UAV-aided WSNs.

\section{Contribution and organization}

The contributions of the paper are summarized as follows:

(i) As a radical alternative to existing methods, we propose an anti-jamming detector leveraging on the structure of the jamming signal rather than treating it as random noise. This allows one to greatly reduce the negative impact of mobile jamming. At a high-level, such an idea is akin to multiuser/multiantenna detection [36], but at a low-level it is significantly different, since the mobile 


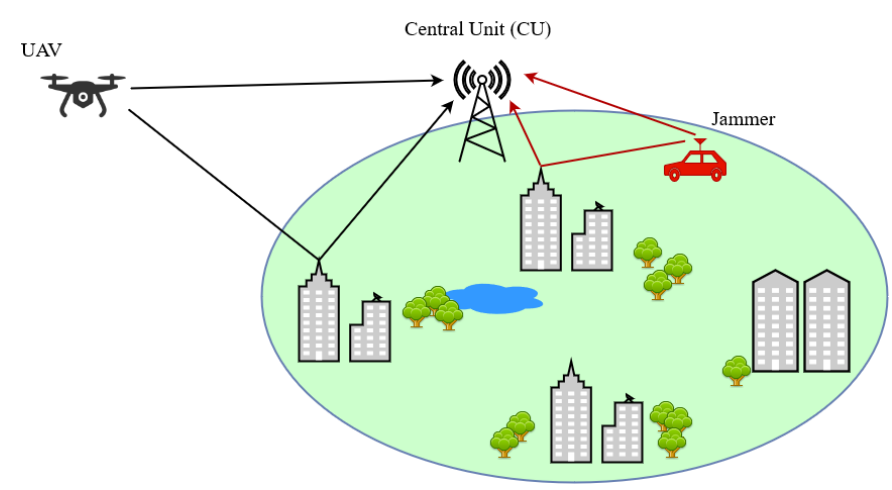

Fig. 1. UAV-aided WSN for smart city applications (downloading phase) in a multipath scenario: the UAV transmits data gathered from ground sensor/meter devices to a $\mathrm{CU}$ in the presence of a smart mobile jammer, whose aim is to degrade the UAV-to-CU transmission.

jammer is not a constituent part of the network and, thus, its transmitter cannot be designed to ease separation of the UAV and jamming signals at the receiver.

(ii) We propose blind algorithms based on ACS properties to estimate the channel parameters of both the UAV and jamming transmissions, by developing a simple rule unveiling the association between the estimated parameters and the two superimposed signals. The developed estimators extend and generalize previously-proposed ACS-based estimation techniques [37]-[41] to doublyselective channels.

(iii) The performances of the proposed channel estimation and detection methods are validated using customary metrics. It is demonstrated that the combination of jamming cancellation for the detection and ACS-based estimation of channel parameters confers robustness against a mobile jamming attack in UAV-based WSNs, under different mobility scenarios of the jammer.

The paper is organized as follows (the main notations are reported in Table I). A reference framework and a model of the considered anti-jamming communication system is described in Section II. The proposed ACS-based estimators of the relevant channel parameters are developed in Section IV. Numerical results are reported in Section V. Finally, the main results obtained in the paper are summarized in Section VI.

\section{THE REFERENCE FRAMEWORK}

With reference to Fig. 1, we focus on the downloading phase where the UAV transmits to the $\mathrm{CU}$, over a dedicated $\mathrm{A} 2 \mathrm{G}$ link, the data collected by the sensors and meters disseminated around the city (the proposed anti-jamming strategy can be used in the uploading phase with minor modifications). An hostile mobile terminal, referred to as the jammer, is trying to disrupt the communication link between the UAV and the CU, by sending jamming signals. To cope with the time-dispersive nature of the channel, the UAV employs OFDM transmission with OFDM symbol period $T$ and sampling period $T_{\mathrm{c}}$.

The UAV transmits data to the CU in a mobility-based fashion aimed at improving the physical layer security [43] of the link. Specifically, we will show that nonzero radial velocities between the UAV and the CU allow to gain robustness against a mobile jamming attack by exploiting their different features in the Doppler domain.

Also the jammer is mobile, e.g., the jamming transmitter can be mounted on a UAV or inside a terrestrial vehicle (as depicted in Fig. 1). From its viewpoint, mobility allows the jammer to achieve two desirable goals: (i) to move quickly from one point to another of the city in order to jam multiple CUs; (ii) to drastically reduce its probability of localization.

\section{A. Basic assumptions}

In this subsection, we state and discuss all the assumptions that are subsequently used throughout the paper.

A1): The jammer is able to replicate almost perfectly the modulation format of the UAV, by injecting in the air random symbols that are independent of those transmitted by the UAV. Compared to the simpler case when the jammer just sends a high-power unmodulated carrier to corrupt the symbol stream of the UAV transmission, the considered attack is harder to be detected using network monitoring tools, since such tools will sense legitimate traffic on the medium [12].

A2): The independent symbol streams $\left\{s_{\mathrm{U}}[n]\right\}$ and $\left\{s_{\mathrm{J}}[n]\right\}$ emitted by the UAV and the mobile jammer $(n \in \mathbb{Z})$, respectively, are modeled as sequences of zero-mean unitvariance independent and identically distributed (i.i.d.) complex noncircular random variables, with second-order moments $\mathbb{E}\left(s_{\mathrm{U}}^{2}[n]\right) \neq 0$ and $\mathbb{E}\left(s_{\mathrm{J}}^{2}[n]\right) \neq 0$, respectively. ${ }^{1}$ Real-valued modulation schemes, such as pulse amplitude modulation (PAM) and binary phase-shift keying (BPSK), naturally exhibit such noncircular statistical features. Furthermore, modulations with quadrature offset, e.g., offset quadrature phase-shift keying (OQPSK), minimum shift keying (MSK) and variants, are noncircular, too: they are attractive for systems using nonlinear amplifiers since the modulated signal is less sensitive to distortion during symbol transitions. Extension of the proposed anti-jamming strategy to circular modulation schemes (i.e., when $\left.\mathbb{E}\left(s_{\mathrm{U}}^{2}[n]\right)=\mathbb{E}\left(s_{\mathbf{J}}^{2}[n]\right)=0\right)$ is outlined in Section III (see Remark 1) and Section IV (see Remark 2).

A3): The signals transmitted by the UAV and the mobile jammer propagate through doubly-selective channels [45], with maximum delay spread $\Delta_{\mathrm{TX}}$ (its reciprocal is proportional to the coherence bandwidth) and Doppler spread $D_{\mathrm{TX}}$ (its reciprocal is proportional to the coherence time) [46], for $\mathrm{TX} \in\{\mathrm{U}, \mathrm{J}\}$. We assume underspread channels, i.e., $\Delta_{\mathrm{U}} D_{\mathrm{U}}<1$ and $\Delta_{\mathrm{J}} D_{\mathrm{J}}<1$, which is a valid assumption for most radio channels [47].

A4): The CU receiver has been previously locked to the multipath component at (approximately) the minimum delay $\tau_{\min }$ between the UAV-to-CU and jammer-to-CU channels, in which case the parameter $\Delta_{\max } \triangleq \max \left(\Delta_{\mathrm{U}}, \Delta_{\mathrm{J}}\right)$ characterizes the time spreading of both channels.

\footnotetext{
${ }^{1}$ Throughout the paper, the subscripts $\mathrm{U}$ and $\mathrm{J}$ indicate parameters referring to the UAV and the jammer, respectively.
} 
A5): The carrier frequency is much larger than the signaling bandwidth. In this case, the shrinking or dilation of the signaling waveform [48, Ch. 7] can be ignored, and the effects of motion are accurately captured by Doppler shifts.

A6): During the downloading phase, the UAV flies at a sufficiently high altitude such that the UAV and the CU have a line-of-sight (LoS) connection with probability 1.

A7): For blind identification of all the channel parameters (see Section IV), all the Doppler shifts of the UAV-to-CU and jammer-to-CU transmissions are different and all their pairs have different sums - a technical condition that is satisfied with probability 1 in real scenarios.

A8): If we denote with $\psi_{\text {DAC }}(t)$ the impulse response of the digital-to-analog converter (DAC) and with $\psi_{\mathrm{ADC}}(t)$ the impulse response of the (anti-aliasing) filter at the input of the analog-to-digital converter (ADC) at the $\mathrm{CU}$, the impulse response $\psi(t) \triangleq \psi_{\mathrm{DAC}}(t) * \psi_{\mathrm{ADC}}(t)$ of the cascade of the DAC interpolation filter and the ADC antialiasing filter obeys $\psi(t) \equiv 0$ for $t \notin\left[0, \Delta_{\text {filter }}\right)$. The pulse $\psi(t)$ and its finite duration $\Delta_{\text {filter }}$ are known at the $\mathrm{CU}$ and $\Delta_{\text {filter }}+\Delta_{\max }<T$ for multicarrier systems (see also A4).

A9): After filtering, the noise observed at the receiver is a zero-mean complex circular white Gaussian random process with power $\sigma_{w}^{2}$.

A10): The OFDM cyclic prefix (CP) length is sufficient to accommodate the overall delay spread, i.e., $L_{\mathrm{cp}} \geq\left(\Delta_{\text {filter }}+\right.$ $\left.\Delta_{\max }\right) / T_{\mathrm{c}}$.

\section{B. Signal models}

According to A1, the flying UAV and the mobile jammer employ $M$ orthogonal subcarriers.

Let $s_{\mathrm{TX}}^{[m]}[\ell] \triangleq s_{\mathrm{TX}}[\ell M+m]$ represent the symbol transmitted in the $\ell$ th data block on the $m$ th subcarrier (see A2), with TX $\in\{\mathrm{U}, \mathrm{J}\}$, the generic symbol vector $\mathbf{s}_{\mathrm{TX}}[n] \triangleq\left(s_{\mathrm{TX}}^{[0]}[n], s_{\mathrm{TX}}^{[1]}[n], \ldots, s_{\mathrm{TX}}^{[M-1]}[n]\right)^{\mathrm{T}} \in \mathbb{C}^{M}$ is subject to IDFT and CP insertion, thus yielding $\mathbf{u}_{\mathrm{TX}}[n] \triangleq$ $\left(u_{\mathrm{TX}}^{[0]}[n], u_{\mathrm{TX}}^{[1]}[n], \ldots, u_{\mathrm{TX}}^{[P-1]}[n]\right)^{\mathrm{T}}=\mathbf{I}_{\mathrm{cp}} \mathbf{W}_{M} \mathbf{s}_{\mathrm{TX}}[n]$, where $\mathbf{I}_{\mathrm{cp}} \in \mathbb{R}^{P \times M}$ models the insertion of a CP of length $L_{\mathrm{cp}}$, with $P \triangleq M+L_{\mathrm{cp}}$ and $\mathbf{W}_{M}$ is the $M$-point IDFT.

The data vector $\mathbf{u}_{\mathrm{TX}}[n]$ undergoes parallel-to-serial $(\mathrm{P} / \mathrm{S})$ conversion, and the resulting sequence $\left\{u_{\mathrm{TX}}[\ell]\right\}$ feeds the DAC, operating at rate $1 / T_{\mathrm{c}}=P / T$, where $u_{\mathrm{TX}}[\ell P+q]=$ $u_{\mathrm{TX}}^{[q]}[\ell]$, for $q \in\{0,1 \ldots, P-1\}$. The baseband transmitted continuous-time signal at the output of the DAC is given, for $\mathrm{TX} \in\{\mathrm{U}, \mathrm{J}\}$, by

$$
x_{\mathrm{TX}}(t)=\sqrt{2 \mathcal{P}_{\mathrm{TX}}} \sum_{\ell=-\infty}^{+\infty} \sum_{q=0}^{P-1} u_{\mathrm{TX}}^{[q]}[\ell] \psi_{\mathrm{DAC}}\left(t-q T_{\mathrm{c}}-\ell T\right)
$$

where $\mathcal{P}_{\text {TX }}$ controls the average transmission radio-frequency power and we recall that $\psi_{\mathrm{DAC}}(t)$ denotes the impulse response of the DAC.
The signal (1) is up-converted and transmitted on the air. Under A3, A4, and A5, the baseband received signal at the $\mathrm{CU}$, after filtering, is given by

$$
\begin{aligned}
y(t)=\sum_{k=1}^{K_{\mathrm{U}}} A_{\mathrm{U}, k} e^{j \theta_{\mathrm{U}, k}} e^{j 2 \pi f_{\mathrm{U}, k} t} x_{\mathrm{U}}\left(t-\tau_{\mathrm{U}, k}\right) \\
\quad+\sum_{k=1}^{K_{\mathrm{J}}} A_{\mathrm{J}, k} e^{j \theta_{\mathrm{J}, k}} e^{j 2 \pi f_{\mathrm{J}, k} t} x_{\mathrm{J}}\left(t-\tau_{\mathrm{J}, k}\right)+w(t)
\end{aligned}
$$

where $K_{\mathrm{TX}}$ is the number of paths between the generic transmitter TX $\in\{\mathbf{U}, \mathbf{J}\}$ and the CU, $A_{\mathrm{TX}, k}>0, \theta_{\mathrm{TX}, k} \in[0,2 \pi)$, $f_{\mathrm{TX}, k} \in\left[-D_{\mathrm{TX}} / 2, D_{\mathrm{TX}} / 2\right]$, and $\tau_{\mathrm{TX}, k} \in\left[0, \Delta_{\mathrm{TX}}\right]$ denote the amplitude, the phase shift, the Doppler (frequency) shift, and delay of the $k$ th path associated with $x_{\mathrm{TX}}(t)$, whereas $w(t)$ is the complex envelope of (filtered) noise, which is statistically independent of $x_{\mathrm{TX}}(t)$. Without loss of generality and according to A6, the LoS component between the UAV and the $\mathrm{CU}$ is assumed to be the first channel path, i.e., the one corresponding to $k=1$ in the leftmost sum in (2). Under A7, the Doppler shifts $f_{\mathrm{U}, 1}, f_{\mathrm{U}, 2}, \ldots, f_{\mathrm{U}, K_{\mathrm{U}}}, f_{\mathrm{J}, 1}, f_{\mathrm{J}, 2}, \ldots, f_{\mathrm{J}, K_{\mathrm{J}}}$ are pairwise-sum different.

The signal (2) is sampled with rate $1 / T_{\mathrm{c}}$ at instants $t_{n, p} \triangleq$ $n T+p T_{\mathrm{c}},{ }^{2}$ for $p \in\{0,1 \ldots, P-1\}$. Let $y^{[p]}[n] \triangleq y\left(t_{n, p}\right)$ be the discrete-time counterpart of (2), one gets

$$
\begin{aligned}
y^{[p]}[n]= & \sum_{k=1}^{K_{\mathrm{U}}} g_{\mathrm{U}, k} e^{j 2 \pi \nu_{\mathrm{U}, k}\left(n+\frac{p}{P}\right)} x_{\mathrm{U}, k}^{[p]}[n] \\
& +\sum_{k=1}^{K_{\mathrm{J}}} g_{\mathrm{J}, k} e^{j 2 \pi \nu_{\mathrm{J}, k}\left(n+\frac{p}{P}\right)} x_{\mathrm{J}, k}^{[p]}[n]+w^{[p]}[n]
\end{aligned}
$$

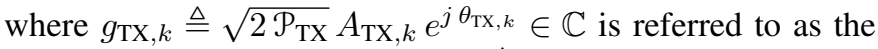
(complex) channel gain and $\nu_{\mathrm{TX}, k} \triangleq f_{\mathrm{TX}, k} T \in[-1 / 2,1 / 2)$ is the normalized Doppler shift, whereas, for $\mathrm{TX} \in\{\mathrm{U}, \mathrm{J}\}$,

$$
\begin{aligned}
x_{\mathrm{TX}, \mathrm{k}}^{[p]}[n] & =\sum_{\ell=n-1}^{n} \sum_{q=0}^{P-1} u_{\mathrm{TX}}^{[q]}[\ell] \\
& \alpha_{\mathrm{TX}, k}\left[(n-\ell) P+(p-q)-d_{\mathrm{TX}, k}\right]
\end{aligned}
$$

where, recalling A8,

$$
\begin{aligned}
\alpha_{\mathrm{TX}, k}[h] & \triangleq \psi\left(h T_{\mathrm{c}}-\chi_{\mathrm{TX}, k}\right) \quad(h \in \mathbb{Z}) \\
\tau_{\mathrm{TX}, k} & =d_{\mathrm{TX}, k} T_{\mathrm{c}}+\chi_{\mathrm{TX}, k}
\end{aligned}
$$

with integer delay $d_{\mathrm{TX}, k} \in\left\{0,1, \ldots,\left\lfloor\Delta_{\mathrm{TX}} / T_{\mathrm{c}}\right\rfloor\right\}$ and fractional delay $\chi_{\mathrm{TX}, k} \in\left[0, T_{\mathrm{c}}\right)$, and, finally, $w^{[p]}[n] \triangleq w\left(t_{n, p}\right)$.

By gathering the obtained samples of the received signal into the vector $\overline{\mathbf{y}}[n] \triangleq\left(y^{[0]}[n], y^{[1]}[n], \ldots, y^{[P-1]}[n]\right)^{\mathrm{T}} \in \mathbb{C}^{P}$ and accounting for (3), we obtain the following vector model

$$
\begin{array}{r}
\overline{\mathbf{y}}[n]=\overline{\mathbf{H}}_{\mathrm{U}, 0}[n] \mathbf{s}_{\mathrm{U}}[n]+\overline{\mathbf{H}}_{\mathrm{U}, 1}[n] \mathbf{s}_{\mathrm{U}}[n-1]+\overline{\mathbf{H}}_{\mathbf{J}, 0}[n] \mathbf{s}_{\mathbf{J}}[n] \\
+\overline{\mathbf{H}}_{\mathbf{J}, 1}[n] \mathbf{s}_{\mathbf{J}}[n-1]+\overline{\mathbf{w}}[n]
\end{array}
$$

\footnotetext{
${ }^{2}$ Hereinafter, it is assumed that $\tau_{\min }=0$ without loss of generality.
} 
TABLE II

LIST OF THE MAIN SYMBOLS USED THROUGHOUT THE PAPER (WITH TX $\in\{\mathrm{U}, \mathrm{J}\}$ ).

\begin{tabular}{|c|c|c|}
\hline & Symbol & Description \\
\hline \multirow{9}{*}{$\begin{array}{l}\text { System } \\
\text { symbols }\end{array}$} & $M$ & number of subcarriers \\
\hline & $L_{\mathrm{cp}}$ & cyclic prefix length \\
\hline & $P$ & OFDM symbol length \\
\hline & $T_{\mathrm{c}}$ & sampling period \\
\hline & $T$ & OFDM symbol period \\
\hline & $f_{0}$ & carrier frequency \\
\hline & $\mathcal{P}_{\text {TX }}$ & average RF transmit power of transmitter TX \\
\hline & $\Delta_{\text {filter }}$ & duration of the ADC impulse response \\
\hline & $\mathbf{s}_{\mathrm{TX}}[n]$ & block of $M$ data symbols transmitted by TX during the $n$th OFDM symbol interval \\
\hline \multirow{11}{*}{$\begin{array}{l}\text { Channel } \\
\text { symbols }\end{array}$} & $\overline{K_{\mathrm{TX}}}$ & number of paths for the TX-to-CU channel \\
\hline & $A_{\mathrm{TX}, k}$ & amplitude of the $k$ th path for the TX-to-CU channel \\
\hline & $\theta_{\mathrm{TX}, k}$ & phase shift of the $k$ th path for the TX-to-CU channel \\
\hline & $f_{\mathrm{TX}, k}$ & Doppler frequency shift of the $k$ th path for the TX-to-CU channel \\
\hline & $\tau_{\mathrm{TX}, k}$ & delay of the $k$ th path for the TX-to-CU channel \\
\hline & $\Delta_{\max }$ & maximum channel time spreading \\
\hline & $g_{\mathrm{TX}, k}$ & channel gain of the $k$ th path for the TX-to-CU channel \\
\hline & $\nu_{\mathrm{TX}, k}$ & normalized Doppler shift of the $k$ th path for the TX-to-CU channel \\
\hline & $d_{\mathrm{TX}, k}$ & integer delay of the $k$ th path for the TX-to-CU channel \\
\hline & $\chi_{\mathrm{TX}, k}$ & fractional delay of the $k$ th path for the TX-to-CU channel \\
\hline & $\mathbf{H}_{\mathrm{TX}}[n]$ & time-varying $M \times M$ TX-to-CU channel matrix after $\mathrm{CP}$ removal \\
\hline
\end{tabular}

where, for $\mathrm{TX} \in\{\mathrm{U}, \mathrm{J}\}$ and $b \in\{0,1\}$,

$$
\overline{\mathbf{H}}_{\mathrm{TX}, b}[n] \triangleq\left[\sum_{k=1}^{K_{\mathrm{TX}}} g_{\mathrm{TX}, k} \mathbf{D}_{\mathrm{TX}, k} \mathbf{T}_{\mathrm{TX}, k, b} e^{j 2 \pi \nu_{\mathrm{TX}, k} n}\right] \boldsymbol{\Omega}
$$

with

$$
\mathbf{D}_{\mathrm{TX}, k} \triangleq \operatorname{diag}\left(1, e^{j \frac{2 \pi}{P} \nu_{\mathrm{TX}, k}}, \ldots, e^{j \frac{2 \pi}{P} \nu_{\mathrm{TX}, k}(P-1)}\right)
$$

whereas $\mathbf{T}_{\mathrm{TX}, k, b} \in \mathbb{R}^{P \times P}$ is a Toeplitz matrix whose $(p+1, q+1)$ th entry is $\alpha_{\mathrm{TX}, k}\left[b P+(p-q)-d_{\mathrm{TX}, k}\right]$, for $p, q \in\{0,1 \ldots, P-1\}, \boldsymbol{\Omega} \triangleq \mathbf{I}_{\mathrm{cp}} \mathbf{W}_{M} \in \mathbb{C}^{P \times M}$, and, according to $\mathrm{A} 9$, the noise vector $\overline{\mathbf{w}}[n] \triangleq$ $\left(w^{[0]}[n], w^{[1]}[n], \ldots, w^{[P-1]}[n]\right)^{\mathrm{T}} \quad \in \quad \mathbb{C}^{P} \quad$ is a zeromean complex circular Gaussian random vector with $\mathbb{E}\left(\overline{\mathbf{w}}\left[n_{1}\right] \overline{\mathbf{w}}^{\mathrm{H}}\left[n_{2}\right]\right)=\sigma_{w}^{2} \delta_{n_{1}-n_{2}} \mathbf{I}_{P}$.

Under A10, after CP removal the received vector (10) gets free from interblock interference (IBI) and assumes the form

$$
\mathbf{y}[n] \triangleq \mathbf{R}_{\mathrm{cp}} \overline{\mathbf{y}}[n]=\mathbf{H}_{\mathrm{U}}[n] \mathbf{s}_{\mathbf{U}}[n]+\mathbf{H}_{\mathbf{J}}[n] \mathbf{s}_{\mathbf{J}}[n]+\mathbf{w}[n]
$$

where matrix $\mathbf{R}_{\mathrm{cp}} \in \mathbb{R}^{M \times P}$ performs CP removal, $\mathbf{H}_{\mathrm{TX}}[n] \triangleq$ $\mathbf{R}_{\mathrm{cp}} \overline{\mathbf{H}}_{\mathrm{TX}, 0}[n] \in \mathbb{C}^{M \times M}$, and, finally, the noise contribution is $\mathbf{w}[n] \triangleq \mathbf{R}_{\mathrm{cp}} \mathbf{\mathbf { w }}[n] \in \mathbb{C}^{M}$. It is noteworthy that both the UAV and the jammer transmissions are adversely affected by intercarrier interference (ICI) due to the presence of Doppler shifts. Indeed, in the absence of a relative motion among the two transmitters and the CU, i.e., when $D_{\mathrm{U}}=D_{\mathrm{J}}=0, \mathbf{H}_{\mathrm{TX}}[n]$ boils down to a time-invariant circulant matrix that can be diagonalized through DFT.

The main symbols introduced in Section II are summarized in Table II. In Section III, we assume that the CU has perfect knowledge of both $\mathbf{H}_{\mathrm{U}}[n]$ and $\mathbf{H}_{\mathrm{J}}[n]$, by deferring to Section IV the challenging problem of how they can be estimated from model (7).

\section{UAV SYMBOL DETECTION WITH ANTI-JAMMING CAPABILITIES}

The signal model (10) describes a multiple-access channel (MAC) [32], [50]-[52], where the CU receives signals from $2 M$ virtual transmitters, each with its own transmit symbol $s_{\mathrm{TX}}^{[p]}[n]$, for $\mathrm{TX} \in\{\mathrm{U}, \mathrm{J}\}$ and $p \in\{0,1 \ldots, M-1\}$. The $\mathrm{CU}$ processes the channel-output vector $\mathbf{y}[n]$ on a symbolby-symbol basis (one-shot detection) to detect the $M$ symbols transmitted by the UAV, which are gathered in the vector $\mathrm{s}_{\mathrm{U}}[n]$.

Two types of strategies can be pursued to detect the UAV symbols. In the former one, for each $p \in\{0,1 \ldots, M-1\}$, the CU detects the UAV symbol $s_{\mathrm{U}}^{[p]}[n]$ by treating the remaining ICI symbols $s_{\mathrm{U}}^{[0]}[n], \ldots, s_{\mathrm{U}}^{[p-1]}[n], s_{\mathrm{U}}^{[p+1]}[n], \ldots, s_{\mathrm{U}}^{[M-1]}[n]$ and jamming symbols $\mathbf{s}_{\mathrm{J}}[n]$ as noise. However, such a strategy leads to poor performance in practice, since the power of ICI plus jamming contribution in (10) is comparable to (or, even, greater than) the power of desired symbol.

In case of strong ICI and jamming, significant performance gains can be achieved by pursuing a different strategy [32], wherein the $\mathrm{CU}$ treats all the inputs as a single vector $\mathbf{s}[n] \triangleq\left(\mathbf{s}_{\mathrm{U}}^{\mathrm{T}}[n], \mathbf{s}_{\mathrm{J}}^{\mathrm{T}}[n]\right)^{\mathrm{T}} \in \mathbb{C}^{2 M}$ and all the symbols are detected jointly. In this strategy, the estimate of the symbol vector $\mathbf{s}_{\mathrm{J}}[n]$ - whose information content is useless to the $\mathrm{CU}$ allows one to eliminate the adverse effects of the jamming symbols on UAV data detection. Unfortunately, optimum (in the minimum-probability-error sense) joint detection [46] entails a computational complexity that grows exponentially with the size of $\mathbf{s}[n]$, and, thus, becomes prohibitive for a large number of subcarriers. Widely-linear $(W L)$ joint detection schemes [53], such as the zero-forcing or minimum-meansquare-error (MMSE) ones, allow one to substantially reduce detection complexity at the expense of (potentially severe) ICI and jamming enhancement.

Herein, we propose the UAV symbol detection procedure with anti-jamming capabilities depicted in Fig. 2. We focus 


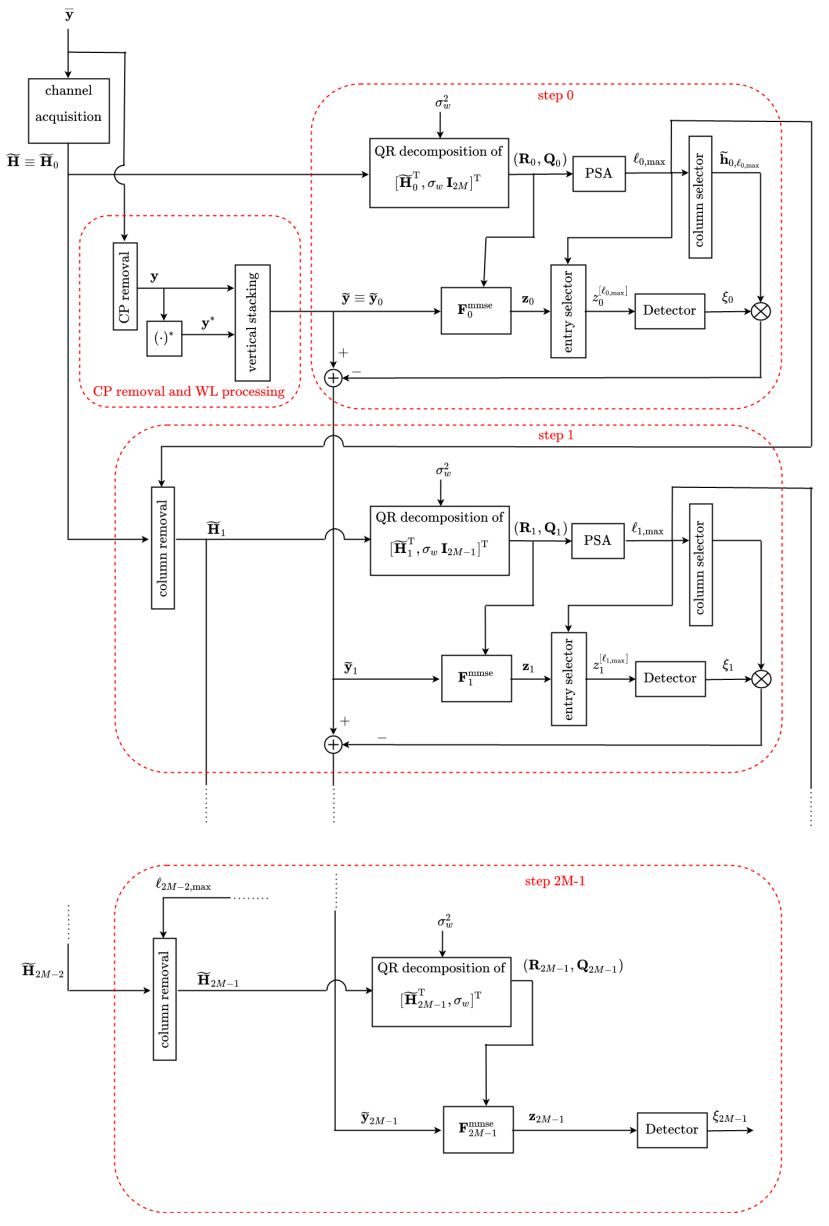

Fig. 2. The proposed anti-jamming reception structure.

on the case where the transmitted symbols are real-valued, i.e., $\mathbf{s}[n]=\mathbf{s}^{*}[n]$ (the developed detection approach can be straightforwardly extended to complex noncircular constellations). To exploit the noncircularity of $\mathbf{s}[n]$, we build the following augmented model for the received signal:

$$
\begin{aligned}
\widetilde{\mathbf{y}}[n] \triangleq\left(\begin{array}{c}
\mathbf{y}[n] \\
\mathbf{y}^{*}[n]
\end{array}\right) & =\underbrace{\left(\begin{array}{cc}
\mathbf{H}_{\mathrm{U}}[n] & \mathbf{H}_{\mathbf{J}}[n] \\
\mathbf{H}_{\mathbf{U}}^{*}[n] & \mathbf{H}_{\mathbf{J}}^{*}[n]
\end{array}\right)}_{\widetilde{\mathbf{H}}[n] \in \mathbb{C}^{(2 M) \times(2 M)}} \mathbf{s}[n]+\underbrace{\left(\begin{array}{c}
\mathbf{w}[n] \\
\mathbf{w}^{*}[n]
\end{array}\right)}_{\widetilde{\mathbf{w}}[n] \in \mathbb{C}^{2 M}} \\
& =\widetilde{\mathbf{H}}[n] \mathbf{s}[n]+\widetilde{\mathbf{w}}[n] .
\end{aligned}
$$

Dropping out the dependence on the block index $n$, the detection procedure consists of $2 M$ steps. As reported in Fig. 2, at the $i$ th step, with $i \in\{0,1, \ldots, 2 M-1\}$, three distinct software-defined functions are executed:

(i) WL-MMSE pre-detection;

(ii) post-sorting algorithm (PSA);

(iii) residual ICI-plus-jamming cancellation, where the initial conditions $\widetilde{\mathbf{y}}_{0}=\widetilde{\mathbf{y}}, \widetilde{\mathbf{H}}_{0}=\widetilde{\mathbf{H}}, \mathbf{s}_{0}=\mathbf{s}$ are set.

The channel state information required by such operations is provided by the estimator discussed in Section IV.

\section{A. WL-MMSE pre-detection}

The WL-MMSE stage provides a preliminary "soft" estimate $\mathbf{z}_{i} \triangleq\left(z_{i}^{[0]}, z_{i}^{[1]}, \ldots, z_{k}^{2 M-i-1]}\right)^{\mathrm{T}} \in \mathbb{C}^{2 M-i}$ of the symbol vector at the $i$ th step, for $i \in\{0,1, \ldots, 2 M-1\}$. In particular, the WL-MMSE detector

$$
\begin{aligned}
\mathbf{F}_{i}^{\mathrm{mmse}} & =\widetilde{\mathbf{H}}_{i}^{\mathrm{H}}\left(\widetilde{\mathbf{H}}_{i} \widetilde{\mathbf{H}}_{i}^{\mathrm{H}}+\sigma_{w}^{2} \mathbf{I}_{2 M}\right)^{-1} \\
& =\left(\widetilde{\mathbf{H}}_{i}^{\mathrm{H}} \widetilde{\mathbf{H}}_{i}+\sigma_{w}^{2} \mathbf{I}_{2 M-i}\right)^{-1} \widetilde{\mathbf{H}}_{i}^{\mathrm{H}}
\end{aligned}
$$

is obtained by minimizing the mean squared error (MSE) $\mathbb{E}\left(\left\|\mathbf{F}_{i} \widetilde{\mathbf{y}}_{i}-\mathbf{s}_{i}\right\|^{2}\right)$ between the output $\mathbf{F}_{i} \widetilde{\mathbf{y}}_{i}$ of the WL detector $\mathbf{F}_{i} \in \mathbb{C}^{(2 M-i) \times(2 M)}$ and the symbol vector $\mathbf{s}_{i}$, where, for $i \in\{1,2, \ldots, 2 M-1\}, \widetilde{\mathbf{y}}_{i} \in \mathbb{R}^{2 M}, \mathbf{s}_{i} \in \mathbb{R}^{2 M-i}$ and $\widetilde{\mathbf{H}}_{i} \in \mathbb{C}^{(2 M) \times(2 M-i)}$ are obtained, respectively, from $\widetilde{\mathbf{y}}_{i-1}$, $\mathbf{s}_{i-1}$, and $\widetilde{\mathbf{H}}_{i-1}$ (see Subsection III-C). The output of the WLMMSE pre-detection stage is

$$
\mathbf{z}_{i}=\mathbf{F}_{i}^{\mathrm{mmse}} \tilde{\mathbf{y}}_{i} .
$$

It is important to note that $\mathbf{F}_{i}^{\mathrm{mmse}} \widetilde{\mathbf{H}}_{i} \neq \mathbf{I}_{2 M-i}$, that is, there are residual ICI and jamming contributions at the output of the WL-MMSE pre-detector.

An equivalent expression of (13) can be obtained by observing that

$$
\widetilde{\mathbf{H}}_{i}^{\mathrm{H}} \widetilde{\mathbf{H}}_{i}+\sigma_{w}^{2} \mathbf{I}_{2 M-i}=\mathbf{G}_{i}^{\mathrm{H}} \mathbf{G}_{i}=\mathbf{R}_{i}^{\mathrm{H}} \mathbf{R}_{i}
$$

with $\mathbf{G}_{i} \triangleq\left(\widetilde{\mathbf{H}}_{i}^{\mathrm{T}}, \sigma_{w} \mathbf{I}_{2 M-i}\right)^{\mathrm{T}} \in \mathbb{C}^{(4 M-i) \times(2 M-i)}$ being fullcolumn rank by construction, i.e., $\operatorname{rank}\left(\mathbf{G}_{i}\right)=2 M-i$, whose economy-size QR decomposition [42] is $\mathbf{G}_{i}=\mathbf{Q}_{i} \mathbf{R}_{i}$, where $\mathbf{Q}_{i} \in \mathbb{C}^{(4 M-i) \times(2 M-i)}$ is a semi-unitary matrix, i.e., $\mathbf{Q}_{i}^{\mathrm{H}} \mathbf{Q}_{i}=\mathbf{I}_{2 M-i}$, and $\mathbf{R}_{i} \in \mathbb{C}^{(2 M-i) \times(2 M-i)}$ is an upper triangular with nonzero diagonal elements. By substituting the QR decomposition of $\mathbf{G}_{i}$ in (12), the pre-detector output (13) can also be written as

$$
\mathbf{z}_{i}=\mathbf{R}_{i}^{-1} \mathbf{Q}_{i}^{\mathrm{H}}\left(\begin{array}{c}
\widetilde{\mathbf{y}}_{i} \\
\mathbf{0}_{2 M-i}
\end{array}\right)
$$

which is more suitable than (13) for the subsequent PSA stage.

Remark 1: When the WL-MMSE criterion is advocated for the soft estimation of complex circular symbols, i.e., $\mathbb{E}\left(s_{\mathrm{U}}^{2}[n]\right)=\mathbb{E}\left(s_{\mathrm{J}}^{2}[n]\right)=0$, eq. (12) boils down to a linear (L) MMSE filter, that is, $\mathbf{F}_{i}^{\mathrm{mmse}}=\left(\mathbf{F}_{i}^{\mathrm{l}-\mathrm{mmse}}, \mathbf{O}_{(2 M-i) \times M}\right)$, where $\mathbf{F}_{i}^{\mathrm{l}-\mathrm{mmse}} \in \mathbb{C}^{(2 M-i) \times M}$ is the standard L-MMSE solution.

\section{B. Post-sorting algorithm (PSA)}

The PSA is basically aimed at individuating the soft symbol estimate having the maximum signal-to-disturbance-plusnoise ratio $(S D N R)$ (or, equivalently, that with the smallest estimation error) at the output of the WL-MMSE filter, where "disturbance" stands for ICI-plus-jamming. In order to introduce the PSA, we preliminarily observe that the post-detection SDNR $\gamma_{i, m}$ for the $(m+1)$ th entry of $\mathbf{s}_{i}$ is given [54] by

$$
\gamma_{i, m}=\frac{1}{\sigma_{w}^{2}\left\{\left(\widetilde{\mathbf{H}}_{i}^{\mathrm{H}} \widetilde{\mathbf{H}}_{i}+\sigma_{w}^{2} \mathbf{I}_{2 M-i}\right)^{-1}\right\}_{m+1, m+1}}-1
$$


for $m \in\{0,1, \ldots, 2 M-i-1\}$. Expression (16) is based on the assumption of perfect knowledge of $\widetilde{\mathbf{H}}_{i}$. On the other hand, when $\widetilde{\mathbf{H}}_{i}$ is replaced by its data-estimated version, eq. (16) turns out to be particularly sensitive to finite-samplesize effects. A certain robustness against channel estimation errors can be gained by resorting to the $\mathrm{QR}$ decomposition of $\mathbf{G}_{i}$ in eq. (14). Since $\mathbf{R}_{i}$ is nonsingular and its inverse $\mathbf{R}_{i}^{-1}$ is another upper triangular matrix [42], one has $\left(\mathbf{R}_{i}^{\mathrm{H}} \mathbf{R}_{i}\right)^{-1}=$ $\mathbf{R}_{i}^{-1}\left[\mathbf{R}_{i}^{-1}\right]^{\mathrm{H}}$. Therefore, the SDNR (16) can be equivalently written as

$$
\gamma_{i, m}=\frac{1}{\sigma_{w}^{2} \sum_{\ell=m}^{2 M-i-1}\left|\left\{\mathbf{R}_{i}^{-1}\right\}_{m+1, \ell+1}\right|^{2}}-1
$$

for $m \in\{0,1, \ldots, 2 M-i-1\}$. We underline that (16) and (17), as well as (13) and (15), are equivalent only when $\widetilde{\mathbf{H}}_{i}$ is perfectly known, whereas they give rise to different values if $\widetilde{\mathbf{H}}_{i}$ is replaced by its data-estimated counterpart. We will resort to (17) for the PSA stage since it is less sensitive to channel estimation errors. The WL-MMSE soft estimate with the largest SDNR corresponds to the $\left(\ell_{i, \max }+1\right)$ th entry $z_{i}^{\left[\ell_{i, \max }\right]}$ of $\mathbf{z}_{i}$, with

$$
\ell_{i, \max }=\arg \max _{\ell \in\{0,1, \ldots, 2 M-i-1\}} \gamma_{i, \ell} .
$$

The "best" soft estimate $z_{i}^{\left[\ell_{i, \max }\right]}$ is then quantized to the nearest (in Euclidean distance) information symbol to form the "hard" estimate $\xi_{i}=\mathcal{Q}\left(z_{i}^{\left[\ell_{i}, \max \right]}\right)$, where $\mathcal{Q}(\cdot)$ is the minimum-distance decision function. It is noteworthy that $\xi_{i}$ may be either the estimate of a symbol transmitted by the UAV or by the jammer.

\section{Residual ICI-plus-jamming cancellation}

The cancellation process reduces the residual ICI and jamming contributions so as to achieve better performance. The basic idea is to cancel out the contribution of $\xi_{i}$ to the remaining symbols, for $i \in\{0,1, \ldots, 2 M-1\}$. Starting from $\xi_{i}$, the canceler executes the cancellation step

$$
\widetilde{\mathbf{y}}_{i+1}=\widetilde{\mathbf{y}}_{i}-\xi_{i} \widetilde{\mathbf{h}}_{i, \ell_{i, \max }}
$$

where $\widetilde{\mathbf{h}}_{i, \ell_{i, \max }} \in \mathbb{C}^{2 M}$ denotes the $\left(\ell_{i, \max }+1\right)$ th column of $\widetilde{\mathbf{H}}_{i}$. In the case of correct detection, the updated version of the received vector reads as

$$
\widetilde{\mathbf{y}}_{i+1}=\widetilde{\mathbf{H}}_{i+1} \mathbf{s}_{i+1}+\widetilde{\mathbf{w}}
$$

where $\widetilde{\mathbf{H}}_{i+1} \in \mathbb{C}^{(2 M) \times(2 M-i-1)}$ is obtained from $\widetilde{\mathbf{H}}_{i}$ by removing its $\left(\ell_{i, \max }+1\right)$ th column $\widetilde{\mathbf{h}}_{i, \ell_{i, \max }}$ and, similarly, $\mathbf{s}_{i+1} \in \mathbb{R}^{2 M-i-1}$ is obtained from $\mathbf{s}_{i}$ by removing its $\left(\ell_{i, \max }+1\right)$ th entry.

\section{Computational complexity}

The proposed UAV symbol detection procedure with antijamming capabilities is summarized as Algorithm 1 at the top of this page. The computational complexity of Algorithm 1 is mainly dominated by the QR decomposition of the partitioned matrix $\mathbf{G}_{i}=\left(\widetilde{\mathbf{H}}_{i}^{\mathrm{T}}, \sigma_{w} \mathbf{I}_{2 M-i}\right)^{\mathrm{T}} \in \mathbb{C}^{(4 M-i) \times(2 M-i)}$, which

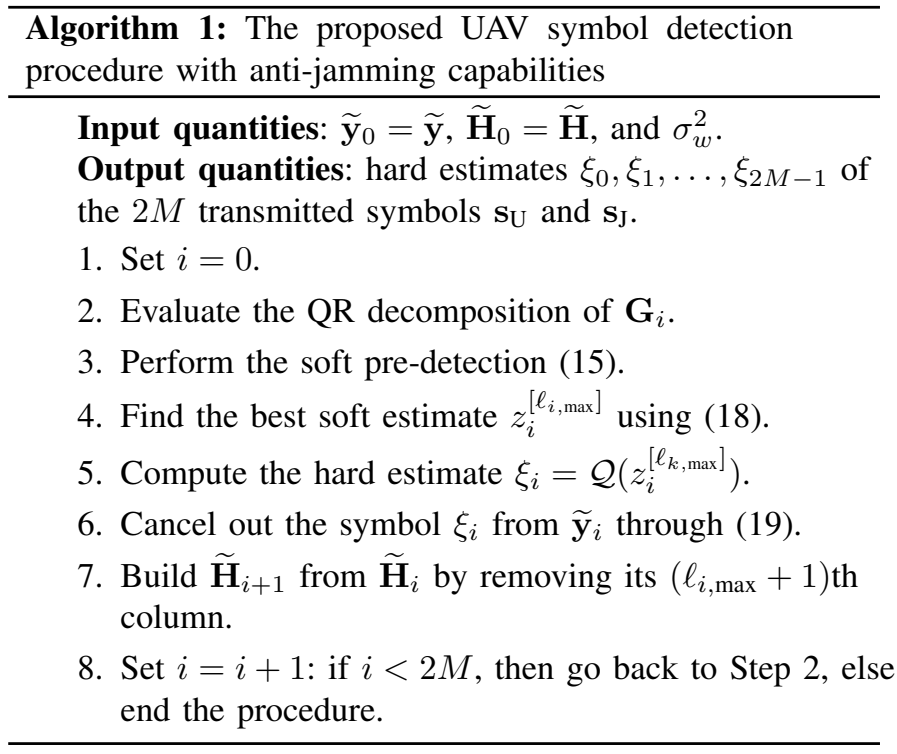

involves $\mathcal{O}\left[(4 M-i)(2 M-i)^{2}\right]$ floating point operations [55] if computed from scratch, for $i \in\{0,1, \ldots, 2 M-1\}$. However, for $i \in\{1,2, \ldots, 2 M-1\}$, the matrix $\mathbf{G}_{i}$ is obtained by deleting a row and a column from $\mathbf{G}_{i-1}$ (socalled downdating operations). Algorithms for downdating the QR decomposition are presented in [55]: they can be modified in a straightforward fashion to reduce the computational complexity of Algorithm 1, by capitalizing on the fact that the QR decomposition behaves nicely under partitioning.

To obtain an estimate of the blocks of symbols transmitted by the UAV during the downloading phase, one has to build the time-varying matrix $\widetilde{\mathbf{H}}[n]$ in (11) and, then, implement Algorithm 1 for each symbol interval $n$ in the downloading time window. Alternatively, one might resort to the frequencydomain representation of such a linear time-variant filtering process [56], which allows one to implement Algorithm 1 by means of linear time-invariant filtering of frequency-shifted versions of the received vector $\tilde{\mathbf{y}}[n]$ in (11), thus reducing run-time complexity (in terms of floating point operations, subscripting, and memory traffic).

\section{ESTIMATION OF CHANNEL PARAMETERS}

The basic assumption underlying the anti-jamming detection strategy developed in Section III is the knowledge of the channel matrices $\mathbf{H}_{\mathrm{U}}[n]$ and $\mathbf{H}_{\mathrm{J}}[n]$. Acquisition of channel state information under a mobile jamming attack is a challenging task, since channel estimation in current wireless data communications standards is mainly based on training sequences sent from the transmitter to the receiver. Such training-based channel estimation algorithms have been designed only to be resilient to non-malicious interference and noise [33]. A jammer can exploit this weakness and efficiently use its power budget in order to significantly contaminate the output of the channel estimator.

Henceforth, we propose a blind solution for estimating $\mathbf{H}_{\mathrm{U}}[n]$ and $\mathbf{H}_{\mathrm{J}}[n]$, which does not rely on training sequences, 
but instead exploits the unique ACS properties of both the UAV and jamming signals. To this aim, for the sake of design, we fix the number of paths $K_{\mathrm{U}}$ between the UAV and the CU to a value large enough to capture all the dominant impinging waves of the UAV transmission. ${ }^{3}$ Instead of estimating the channels $\mathbf{H}_{\mathrm{U}}[n]$ and $\mathbf{H}_{\mathrm{J}}[n]$ (communication theory viewpoint), we choose herein to estimate the parameters characterizing such channels, i.e., $g_{\mathrm{TX}, k}, f_{\mathrm{TX}, k}$ or, equivalently, $\nu_{\mathrm{TX}, k}$, and $\tau_{\mathrm{TX}, k}$, for $k \in\left\{1,2, \ldots, K_{\mathrm{TX}}\right\}$ and $\mathrm{TX} \in\{\mathrm{U}, \mathrm{J}\}$, by following an array signal processing approach.

Specifically, we rely on the pioneering works [37], [38], wherein several reliable estimation methods based on signal cyclostationarity have been developed for time-difference-ofarrival of a single signal of interest (SOI), such as those based on cyclic cross-correlation, spectral correlation ratio, and spectral coherence alignment. Estimation of both power and timing parameters of multiple SOIs has been discussed in [39]. However, the approaches in [37]-[39] are targeted at flat-flat channels, for which there exists a single path only, i.e., $K_{\mathrm{U}}=K_{\mathrm{J}}=1$ in (2), and the Doppler shift can be assumed to be zero over the observation time interval, i.e., $f_{\mathrm{U}, 1}=f_{\mathrm{J}, 1}=0$ in (2). The basic methodologies developed in [37]-[39] can be extended to time-varying channels, by capitalizing on the fact that Doppler shifts change the spectral correlation property of the transmitted signals [40], [41, Ch. 8]. However, it is assumed in such studies that the channel is time selective but frequency nonselective (or frequency flat), i.e., $K_{\mathrm{U}}=K_{\mathrm{J}}=1$ in (2), which is a restrictive assumption for smart city applications, where the channel might be non-flat in both time and frequency.

In the forthcoming subsections, we exploit the ACS properties of the received signal to blindly estimate channel gains, Doppler shifts, and time delays of both the UAV and jamming transmissions, by generalizing the methods developed in [37][41] to the case of doubly-selective channels. The starting point of our estimation procedure is signal model (7), i.e., the entire OFDM block (including the CP) is processed for channel estimation purposes, by also taking advantage of IBI.

\section{A. Second-order wide-sense statistical characterization of the received signal}

The ACS features of the received signal (7) stem from the fact that $\overline{\mathbf{H}}_{\mathrm{TX}, b}[n]$ in (8) is a discrete-time almost-periodic (AP) matrix [57] with (possibly) incommensurate frequencies belonging to the (order) set

$$
\mathcal{A}_{\mathrm{TX}} \triangleq\left\{\nu_{\mathrm{TX}, 1}, \nu_{\mathrm{TX}, 2}, \ldots, \nu_{\mathrm{TX}, K_{\mathrm{TX}}}\right\} \subseteq[-1 / 2,1 / 2) .
$$

As a consequence, for $r \in\{-1,0,1\}$, the correlation matrix $\mathbf{R}_{\overline{\mathbf{y y}}}[n, r] \triangleq \mathbb{E}\left(\overline{\mathbf{y}}[n] \overline{\mathbf{y}}^{\mathrm{H}}[n-r]\right) \in \mathbb{C}^{P \times P}$ and the conjugate correlation matrix $\mathbf{R}_{\overline{\mathbf{y y}^{*}}}[n, r] \triangleq \mathbb{E}\left(\overline{\mathbf{y}}[n] \overline{\mathbf{y}}^{\mathrm{T}}[n-r]\right) \in \mathbb{C}^{P \times P}$ are both AP matrices. Accordingly, the multivariate process (7) is said to be second-order wide-sense almost-cyclostationary [34]. To save space, in the sequel, we use the notation

\footnotetext{
${ }^{3}$ Alternatively, classical information theoretic methods [49] for model selection can be used to estimate the exact value of $K_{\mathrm{U}}$ during a secure setup session.
}

$\mathbf{R}_{\overline{\mathbf{y y}}^{(*)}}[n, r] \triangleq \mathbb{E}\left(\overline{\mathbf{y}}[n]\left\{\overline{\mathbf{y}}^{\mathrm{H}}[n-r]\right\}^{(*)}\right) \in \mathbb{C}^{P \times P}$, where the superscript $(*)$ denotes optional complex conjugation: if conjugation is absent, then $\mathbf{R}_{\overline{\overline{y y}^{(*)}}}[n, r] \equiv \mathbf{R}_{\overline{\mathbf{y y}}}[n, r]$; if conjugation is present, then $\mathbf{R}_{\overline{\overline{y y}^{(*)}}}[n, r] \equiv \mathbf{R}_{\overline{\mathbf{y y}}^{*}}[n, r]$.

It can be verified that

$$
\begin{aligned}
& \mathbf{R}_{\overline{\mathbf{y y}}^{(*)}}[n, r]=\sum_{k_{1}=1}^{K_{\mathrm{U}}} \sum_{h_{1}=1}^{K_{\mathrm{U}}} \boldsymbol{\Xi}_{\mathrm{U}^{(*)}, k_{1}, h_{1}}[r] e^{j 2 \pi\left(\nu_{\mathrm{U}, k_{1}}-(-) \nu_{\mathrm{U}, h_{1}}\right) n} \\
& +\sum_{k_{2}=1}^{K_{\mathrm{J}}} \sum_{h_{2}=1}^{K_{\mathrm{J}}} \boldsymbol{\Xi}_{\mathbf{J}^{(*)}, k_{2}, h_{2}}[r] e^{j 2 \pi\left(\nu_{\mathrm{J}, k_{2}}-(-) \nu_{\mathrm{J}, h_{2}}\right) n}+\mathbf{R}_{\overline{\mathbf{w}}}(*)[r]
\end{aligned}
$$

where $(-)$ is an optional minus sign linked to $(*)$ : if conjugate is absent, then $-(-)=-$; if conjugate is present, then $-(-)=+$. Moreover, the matrices in (22) are defined in (23)(25) at the top of the next page, for $k, h \in\left\{1,2, \ldots, K_{\mathrm{TX}}\right\}$ and $\mathrm{TX} \in\{\mathrm{U}, \mathbf{J}\}, \mathbf{R}_{\overline{\overline{\mathbf{w}}^{(*)}}}[r] \triangleq \mathbb{E}\left(\overline{\mathbf{w}}[n]\left\{\overline{\mathbf{w}}^{\mathrm{H}}[n-r]\right\}^{(*)}\right) \in \mathbb{C}^{P \times P}$ is given by $\mathbf{R}_{\overline{\mathbf{w w}}}[r]=\sigma_{w}^{2} \mathbf{I}_{P} \delta_{r}$ when conjugation is absent, whereas it boils down to $\mathbf{R}_{\overline{\mathbf{w w}^{*}}}[r]=\mathbf{O}_{P \times P}$, for any $r \in \mathbb{Z}$, when conjugation is present. The frequency set $\mathcal{A}_{\overline{\overline{y y}^{(*)}}}$ of the AP matrix (22) can be written as reported in (26) at the top of the next page, which collects the pairwise difference of the Doppler shifts in $\mathcal{A}_{\mathrm{U}}$ and $\mathcal{A}_{\mathrm{J}}$ in the case of the correlation matrix $\mathbf{R}_{\overline{\mathbf{y y}}}[n, r]$, whereas it gathers the pairwise sum of the Doppler shifts in $\mathcal{A}_{\mathrm{U}}$ and $\mathcal{A}_{\mathrm{J}}$ when the conjugate correlation matrix $\mathbf{R}_{\overline{\mathbf{y y}}}[n, r]$ is of concern.

Under mild regularity conditions, the AP matrix (22) can be equivalently written in terms of the generalized Fourier series expansion [57] as follows

$$
\mathbf{R}_{\overline{\mathbf{y y}}(*)}[n, r]=\sum_{\alpha \in \mathcal{A}_{\overline{\mathbf{y y}}(*)}} \mathbf{R}_{\overline{\mathbf{y y}}(*)}^{\alpha}[r] e^{j 2 \pi \alpha n}
$$

where, for $r \in\{-1,0,1\}$,

$$
\begin{aligned}
\mathbf{R}_{\overline{\mathbf{y}} \bar{y}^{(*)}}^{\alpha}[r] & \triangleq\left\langle\mathbf{R}_{\overline{\mathbf{y y}}(*)}[n, r] e^{-j 2 \pi \alpha n}\right\rangle \\
& =\lim _{N \rightarrow+\infty} \frac{1}{2 N+1} \sum_{n=-N}^{N} \mathbf{R}_{\overline{\overline{y y}^{(*)}}}[n, r] e^{-j 2 \pi \alpha n}
\end{aligned}
$$

is the $\alpha$ th generalized Fourier series coefficient of the timevarying correlation matrix $\mathbf{R}_{\overline{\mathbf{y y}}{ }^{(*)}}[n, r]$, which is also referred to as the cyclic correlation matrix (CCM) at cycle frequency $\alpha$ when conjugation is absent, whereas it is called the conjugate cyclic correlation matrix (CCCM) at cycle frequency $\alpha$ when conjugation is present. It can be verified that, for $r \in\{-1,0.1\}$,

$$
\mathbf{R}_{\overline{\mathbf{y y}}^{(*)}}^{\alpha}[r]= \begin{cases}\boldsymbol{\Xi}_{\mathrm{U}^{(*)}, k_{1}, h_{1}}[r], & \text { for } \alpha=\alpha_{\mathrm{U}^{(*)}, k_{1}, h_{1}} \\ & k_{1}, h_{1} \in\left\{1,2, \ldots, K_{\mathrm{U}}\right\} \\ \boldsymbol{\Xi}_{\mathbf{J}^{(*)}, k_{2}, h_{2}}[r], & \text { for } \alpha=\alpha_{\mathbf{J}^{(*)}, k_{2}, h_{2}} \\ & k_{2}, h_{2} \in\left\{1,2, \ldots, K_{\mathrm{J}}\right\}\end{cases}
$$




$$
\begin{aligned}
& \boldsymbol{\Xi}_{\mathrm{TX}(*), k, h}[-1] \triangleq g_{\mathrm{TX}, k}\left(g_{\mathrm{TX}, h}^{*}\right)^{(*)}\left(e^{-j 2 \pi \nu_{\mathrm{TX}, h}}\right)^{(*)} \mathbf{D}_{\mathrm{TX}, k} \mathbf{T}_{\mathrm{TX}, k, 0} \boldsymbol{\Omega}\left(\boldsymbol{\Omega}^{\mathrm{H}} \mathbf{T}_{\mathrm{TX}, h, 1}^{\mathrm{H}} \mathbf{D}_{\mathrm{TX}, h}^{*}\right)^{(*)} \in \mathbb{C}^{P \times P} \\
& \boldsymbol{\Xi}_{\mathrm{TX}(*), k, h}[0] \triangleq g_{\mathrm{TX}, k}\left(g_{\mathrm{TX}, h}^{*}\right)^{(*)} \mathbf{D}_{\mathrm{TX}, k}\left[\mathbf{T}_{\mathrm{TX}, k, 0} \boldsymbol{\Omega}\left(\boldsymbol{\Omega}^{\mathrm{H}} \mathbf{T}_{\mathrm{TX}, h, 0}^{\mathrm{H}}\right)^{(*)}+\mathbf{T}_{\mathrm{TX}, k, 1} \boldsymbol{\Omega}\left(\boldsymbol{\Omega}^{\mathrm{H}} \mathbf{T}_{\mathrm{TX}, h, 1}^{\mathrm{H}}\right)^{(*)}\right]\left(\mathbf{D}_{\mathrm{TX}, h}^{*}\right)^{(*)} \in \mathbb{C}^{P \times P} \\
& \boldsymbol{\Xi}_{\mathrm{TX}^{(*)}, k, h}[1] \triangleq g_{\mathrm{TX}, k}\left(g_{\mathrm{TX}, h}^{*}\right)^{(*)}\left(e^{j 2 \pi \nu_{\mathrm{TX}, h}}\right)^{(*)} \mathbf{D}_{\mathrm{TX}, k} \mathbf{T}_{\mathrm{TX}, k, 1} \boldsymbol{\Omega}\left(\boldsymbol{\Omega}^{\mathrm{H}} \mathbf{T}_{\mathrm{TX}, h, 0}^{\mathrm{H}} \mathbf{D}_{\mathrm{TX}, h}^{*}\right)^{(*)} \in \mathbb{C}^{P \times P}
\end{aligned}
$$

$$
\begin{aligned}
\mathcal{A}_{\overline{\mathbf{y y}}(*)}=\left\{\alpha_{\mathrm{U}^{(*)}, k_{1}, h_{1}}=\nu_{\mathrm{U}, k_{1}}-(-) \nu_{\mathrm{U}, h_{1}}(\bmod 1), \text { for } k_{1}, h_{1} \in\left\{1,2, \ldots, K_{\mathrm{U}}\right\}\right\} \\
\cup\left\{\alpha_{\mathbf{J}^{(*)}, k_{2}, h_{2}}=\nu_{\mathrm{J}, k_{2}}-(-) \nu_{\mathrm{J}, h_{2}}(\bmod 1), \text { for } k_{2}, h_{2} \in\left\{1,2, \ldots, K_{\mathrm{J}}\right\}\right\}
\end{aligned}
$$

whereas $\mathbf{R}_{\overline{\mathbf{y y}}^{(*)}}^{\alpha}[r] \equiv \mathbf{O}_{P \times P}$ if $\alpha \notin \mathcal{A}_{\overline{\mathbf{y y}}^{(*)}}{ }^{4}$. The proposed estimation approaches rely only on the conjugate secondorder statistics of the almost-cyclostationary random process (7). In principle, the knowledge of the correlation matrices $\mathbf{R}_{\overline{\mathbf{y y}}}[n,-1], \mathbf{R}_{\overline{\mathbf{y y}}}[n, 0]$, and $\mathbf{R}_{\overline{\mathbf{y y}}}[n, 1]$ can also be exploited to improve estimation accuracy and/or relax some assumptions. ${ }^{5}$ However, these enhancements are not pursued herein.

Remark 2: When the transmitted symbols are circular, the conjugate correlation matrix of the received signal (7) is identically zero, i.e., $\mathbf{R}_{\overline{\mathbf{y y}}}[n, r] \equiv \mathbf{O}_{P \times P}$. In this case, as an alternative, the proposed estimation algorithms can be suitably modified to exploit higher-order cyclostationarity properties of the received signal [61].

\section{B. Estimation of Doppler shifts}

Estimation of Doppler shifts $f_{\mathrm{TX}, k}$ is equivalent to that of $\nu_{\mathrm{TX}, k}$, for $k \in\left\{1,2, \ldots, K_{\mathrm{TX}}\right\}$ and $\mathrm{TX} \in\{\mathrm{U}, \mathrm{J}\}$. Such a knowledge can be acquired by relying on the AP conjugate correlation matrices $\mathbf{R}_{\overline{\mathbf{y y}^{*}}}[n,-1], \mathbf{R}_{\overline{\mathbf{y y}}}{ }^{*}[n, 0]$, and $\mathbf{R}_{\overline{\mathbf{y y}^{*}}}[n, 1]$ in (22), where the cardinality of the frequency set $\mathcal{A}_{\overline{\mathbf{y y}}}$ * defined in (26) is equal to

$$
L_{\mathrm{a}} \triangleq\left[K_{\mathrm{U}}\left(K_{\mathrm{U}}+1\right)+K_{\mathrm{J}}\left(K_{\mathrm{J}}+1\right)\right] / 2
$$

since its elements are different by assumption. In the case of frequency-flat channels, i.e., $K_{\mathrm{U}}=K_{\mathrm{J}}=1$, it follows that $\mathcal{A}_{\overline{\mathbf{y y}}{ }^{*}}=\left\{2 \nu_{\mathrm{U}, 1}, 2 \nu_{\mathrm{J}, 1}\right\}$. The complication due to the presence of multipath stems from the fact that, when $K_{\mathrm{U}}>1$ and $K_{\mathrm{J}}>1$, the set $\mathcal{A}_{\overline{\mathbf{y y}}}{ }^{*}$ contains also the pairwise sums of the elements of $\mathcal{A}_{\mathrm{U}}$ and $\mathcal{A}_{\mathrm{J}}$, which significantly complicates estimation of the individual Doppler shift sets $\mathcal{A}_{\mathrm{U}}$ and $\mathcal{A}_{\mathrm{J}}$ from the received data.

Herein, we first derive the proposed algorithm to estimate the Doppler shifts under the assumption that $\mathbf{R}_{\overline{\mathbf{y y}}^{*}}^{\alpha}[-1]$, $\mathbf{R}_{\mathbf{y} \mathbf{y}^{*}}^{\alpha}[0]$, and $\mathbf{R}_{\mathbf{y} \mathbf{y}^{*}}^{\alpha}[1]$ are perfectly known; the synthesis of its data-estimated version is briefly discussed at the end of this subsection. Knowledge of the CCCMs $\mathbf{R}_{\mathbf{y y}^{*}}^{\alpha}[-1], \mathbf{R}_{\mathbf{y y}^{*}}^{\alpha}[0]$, and $\mathbf{R}_{\mathbf{y y}^{*}}^{\alpha}$ [1] enables one to blindly retrieve the unknown cycle

\footnotetext{
${ }^{4}$ Eq. (29) holds in the more general case where the noise vector $\overline{\mathbf{w}}[n]$ in (7) is a non-stationary colored random process that does not exhibit almostcyclostationarity at the cycle frequency $\alpha \in \mathcal{A}_{\overline{\mathbf{y y}}(*)}$.

${ }^{5}$ For instance, the assumption that the Doppler shifts are pairwise-sum different might be relaxed by also using the set $\mathcal{A}_{\overline{\mathbf{y y}}}$.
}

frequencies $\left\{\alpha_{\mathrm{U}^{(*)}, k_{1}, h_{1}}\right\}_{k_{1}, h_{1}=1}^{K_{\mathrm{U}}}$ and $\left\{\alpha_{\mathbf{J}^{(*)}, k_{1}, h_{1}}\right\}_{k_{1}, h_{1}=1}^{K_{\mathrm{J}}}$ by defining the one-dimensional objective function:

$$
\begin{gathered}
J(\alpha) \triangleq \sum_{r=-1}^{1}\left\|\mathbf{R}_{\overline{\mathbf{y}} \mathbf{y}^{*}}^{\alpha}[r]\right\|^{2} \\
=\sum_{r=-1}^{1} \operatorname{tr}\left(\mathbf{R}_{\overline{\mathbf{y}}^{*}}^{\alpha}[r]\left\{\mathbf{R}_{\overline{\mathbf{y y}}^{*}}^{\alpha}[r]\right\}^{\mathrm{H}}\right), \\
\quad \text { with } \alpha \in[-1 / 2,1 / 2) .
\end{gathered}
$$

By using differential calculus arguments, it can be analytically shown that $J(\alpha)$ has local maxima in $[-1 / 2,1 / 2)$ at points $\left\{\alpha_{\mathrm{U}^{(*)}, k_{1}, h_{1}}\right\}_{k_{1}, h_{1}=1}^{K_{\mathrm{U}}}$ and $\left\{\alpha_{\mathrm{J}^{(*)}, k_{2}, h_{2}}\right\}_{k_{2}, h_{2}=1}^{K_{\mathrm{J}}}$. Hence, the cycle frequencies of the second-order almost-cyclostationary process (7) can be acquired by searching for the $L_{\mathrm{a}}$ local maxima of (31) over the unit interval $[-1 / 2,1 / 2){ }^{6}$ Starting from the knowledge of $K_{\mathrm{U}}$ and $L_{\mathrm{a}}$, and recalling (30), the number of paths $K_{\mathrm{J}}$ between the jammer and the $\mathrm{CU}$ can be obtained by taking the positive solution of the second-order equation $K_{\mathrm{J}}^{2}+K_{\mathrm{J}}=2 L_{\mathrm{a}}-K_{\mathrm{U}}\left(K_{\mathrm{U}}+1\right)$.

Let $\boldsymbol{\alpha}_{\overline{\overline{y y}^{*}}} \triangleq\left(\alpha_{1}, \alpha_{2}, \ldots, \alpha_{L_{\mathrm{a}}}\right)^{\mathrm{T}} \in[-1 / 2,1 / 2)^{L_{\mathrm{a}}}$ collect the $L_{\mathrm{a}}$ maximum points of $J(\alpha)$ in the interval $[-1 / 2,1 / 2)$, the next step is to infer the Doppler shifts gathered into the two vectors $\nu_{\mathrm{U}} \triangleq\left(\nu_{\mathrm{U}, 1}, \nu_{\mathrm{U}, 2}, \ldots, \nu_{\mathrm{U}, K_{\mathrm{U}}}\right)^{\mathrm{T}} \in[-1 / 2,1 / 2)^{K_{\mathrm{U}}}$ and $\nu_{\mathrm{J}} \triangleq\left(\nu_{\mathrm{J}, 1}, \nu_{\mathrm{J}, 2}, \ldots, \nu_{\mathrm{J}, K_{\mathrm{J}}}\right)^{\mathrm{T}} \in[-1 / 2,1 / 2)^{K_{\mathrm{J}}}$ from the knowledge of $\boldsymbol{\alpha}_{\overline{\mathbf{y y}}^{*}}$. Let $\boldsymbol{\nu}_{\text {ord }} \triangleq\left(\boldsymbol{\nu}_{\mathrm{U}}^{\mathrm{T}}, \boldsymbol{\nu}_{\mathrm{J}}^{\mathrm{T}}\right)^{\mathrm{T}} \in[-1 / 2,1 / 2)^{K}$.

Proposition 4.1: Estimation of $\nu_{\text {ord }}$ from the observed vector $\boldsymbol{\alpha}_{\overline{\mathbf{y y}}}{ }^{*}$ is tantamount to solving the matrix equation

$$
\mathbf{P}_{\overline{\mathbf{y}} \overline{\mathbf{y}}^{*}} \mathbf{B} \nu_{\text {ord }}=\alpha_{\overline{\mathbf{y} \mathbf{y}^{*}}}
$$

with respect to the unknowns $\mathbf{P}_{\overline{\mathbf{y y}}^{*}}$ and $\boldsymbol{\nu}_{\text {ord }}$, where we have defined the matrix $\mathbf{B} \triangleq\left(2 \mathbf{I}_{K}, \boldsymbol{\Gamma}^{\mathrm{T}}\right)^{\mathrm{T}} \in \mathbb{R}^{L_{\mathrm{a}} \times K}$, with

$$
\boldsymbol{\Gamma} \triangleq\left(\begin{array}{ccccc}
1 & 1 & 0 & \ldots & 0 \\
1 & 0 & 1 & \ldots & 0 \\
\vdots & \ldots & \ddots & \ddots & \vdots \\
1 & 0 & 0 & \ldots & 1 \\
0 & 1 & 1 & \ldots & 0 \\
\vdots & \ldots & \ddots & \ddots & \vdots
\end{array}\right) \in \mathbb{R}^{\left(L_{\mathrm{a}}-K\right) \times K}
$$

${ }^{6}$ Acquisition of $L_{\mathrm{a}}$ can be obtained by counting the number of local maxima of $J(\alpha)$ and/or by using classical information theoretic methods for model selection, such as Akaike's and Rissanen's criteria [49]. 
whereas $\mathbf{P}_{\overline{\mathbf{y y}^{*}}} \in \mathbb{R}^{L_{\mathrm{a}} \times L_{\mathrm{a}}}$ is a permutation matrix [42] having exactly one entry in each row and column equal to 1 , and all the other entries equal to 0.

Proof: See Appendix A.

The permutation matrix $\mathbf{P}_{\overline{\mathbf{y y}}}{ }^{*}$ is completely characterized by a vector of integers, where the integer at position $i$ is the column index of the unit element of row $i$ of $\mathbf{P}_{\overline{\mathbf{y y}}}$. Hence, eq. (61) represents a mixed-integer system of $L_{\mathrm{a}}$ linear equations.

System (61) is underdetermined in the unknowns $\mathbf{P}_{\overline{\mathbf{y y}}}$ and $\boldsymbol{\nu}_{\text {ord }}$, and can be solved in the least-squares (LS) sense (see, e.g., [58]) by formulating the following constrained problem:

$$
\arg \min _{\mathbf{P} \in \mathcal{P}}\left\|\mathbf{P} \mathbf{B} \boldsymbol{\nu}-\boldsymbol{\alpha}_{\overline{\mathbf{y}} \bar{y}^{*}}\right\|^{2}
$$

where $\mathcal{P}$ denote the subset of all $L_{\mathrm{a}} \times L_{\mathrm{a}}$ permutation matrices.

For a given permutation matrix $\mathbf{P}$, minimization in (34) with respect to $\nu$ is an overdetermined LS problem, whose solution is given by $\boldsymbol{\nu}_{\mathrm{LS}}=\left(\mathbf{B}^{\mathrm{T}} \mathbf{B}\right)^{-1} \mathbf{B}^{\mathrm{T}} \mathbf{P}^{\mathrm{T}} \boldsymbol{\alpha}_{\overline{\mathbf{y y}}}{ }^{*}$ [58]. Substituting such a LS solution into (34), we obtain the integer linear programming problem:

$$
\arg \max _{\mathbf{P} \in \mathcal{P}}\left(\boldsymbol{\alpha}_{\overline{\mathbf{y}}}^{\mathrm{T}} * \mathbf{P} \boldsymbol{\Pi} \mathbf{P}^{\mathrm{T}} \boldsymbol{\alpha}_{\overline{\mathbf{y y}} *}\right)
$$

where $\boldsymbol{\Pi} \triangleq \mathbf{B}\left(\mathbf{B}^{\mathrm{T}} \mathbf{B}\right)^{-1} \mathbf{B}^{\mathrm{T}} \in \mathbb{R}^{L_{\mathrm{a}} \times L_{\mathrm{a}}}$ is the orthogonal projector onto the range (or column space) of $\mathbf{B}$. Since $\mathbf{P}^{\mathrm{T}}=\mathbf{P}^{-1}$ permutes columns in the same way as the permutation matrix $\mathbf{P}$ permutes rows, the transformation $\boldsymbol{\Pi} \rightarrow \mathbf{P} \boldsymbol{\Pi} \mathbf{P}^{\mathrm{T}}$ permutes the rows and the columns of $\Pi$ in the same way. By virtue of the Rayleigh-Ritz theorem [42], one has

$$
\begin{aligned}
& \boldsymbol{\alpha}_{\overline{\mathbf{y y}}}^{\mathrm{T}} \mathbf{P} \boldsymbol{\Pi} \mathbf{P}^{\mathrm{T}} \boldsymbol{\alpha}_{\overline{\overline{y y}^{*}}} \leq \lambda_{\max }\left(\mathbf{P} \boldsymbol{\Pi} \mathbf{P}^{\mathrm{T}}\right)\left\|\boldsymbol{\alpha}_{\overline{\overline{y y}^{*}}}\right\|^{2} \\
& =\lambda_{\max }(\boldsymbol{\Pi})\left\|\boldsymbol{\alpha}_{\overline{\mathbf{y y}}}\right\|^{2}=\left\|\boldsymbol{\alpha}_{\overline{\mathbf{y y}} \overline{\mathbf{y}}^{*}}\right\|^{2}=\left\|\boldsymbol{\alpha}_{\text {ord }}\right\|^{2}
\end{aligned}
$$

where we have used the facts that the transformation $\Pi \rightarrow$ $\mathbf{P} \Pi \mathbf{P}^{\mathrm{T}}$ does not alter the eigenvalues of $\boldsymbol{\Pi}$ and, additionally, the eigenvalues of an orthogonal projector matrix are 0 or 1 . Taking into account (56)-(58) and remembering that $\mathbf{P}_{\overline{\mathbf{y y}}^{*}}^{\mathrm{T}} \mathbf{P}_{\overline{\mathbf{y y}}^{*}}=\mathbf{I}_{L_{\mathrm{a}}}$, it is readily verified that the cost function in (35) is maximized when $\mathbf{P}=\mathbf{P}_{\overline{\mathbf{y y}}^{*}}$. However, it can be numerically verified that, owing to the particular structure of matrix B in (60), there exist permutation matrices $\mathbf{P} \neq \mathbf{P}_{\overline{\mathbf{y y}}}{ }^{*}$ such that $\mathbf{P} \boldsymbol{\Pi} \mathbf{P}^{\mathrm{T}}=\mathbf{P}_{\overline{\mathbf{y y}}} \boldsymbol{\Pi}_{\mathbf{P}_{\overline{\mathbf{y y}}}}^{\mathrm{T}}$. In a nutshell, the optimization problem (35) admits more than one solution: this result mathematically certifies the fact that the composite mapping $\mathcal{L}_{1} \circ \mathcal{L}_{2}$ is not invertible, as previously announced.

Let $\mathbf{P}_{\mathrm{LS}, 0}, \mathbf{P}_{\mathrm{LS}, 1}, \ldots, \mathbf{P}_{\mathrm{LS}, Q-1}$ denote the $Q$ permutation matrices achieving the same optimal value $\left\|\boldsymbol{\alpha}_{\overline{\mathbf{y y}}}{ }^{*}\right\|^{2}$ in (35), where $\mathbf{P}_{\mathrm{LS}, 0} \triangleq \mathbf{P}_{\overline{\mathbf{y y}}{ }^{*}}$ and $Q$ can be upper bounded as $Q<K$, and consider the corresponding estimates of the Doppler shifts

$$
\begin{aligned}
\boldsymbol{\nu}_{\mathrm{LS}, q}=\left(\mathbf{B}^{\mathrm{T}} \mathbf{B}\right)^{-1} \mathbf{B}^{\mathrm{T}} \mathbf{P}_{\mathrm{LS}, q}^{\mathrm{T}} \boldsymbol{\alpha}_{\overline{\mathbf{y}} \mathbf{y}^{*}} \\
\qquad \\
\quad \text { for } q \in\{0,1, \ldots, Q-1\} .
\end{aligned}
$$

It can be observed by direct inspection that $\boldsymbol{\nu}_{\mathrm{LS}, 0}=\boldsymbol{\nu}_{\text {ord }}$, whereas $\boldsymbol{\nu}_{\mathrm{LS}, 1}, \boldsymbol{\nu}_{\mathrm{LS}, 2}, \ldots, \boldsymbol{\nu}_{\mathrm{LS}, Q-1}$ are different permutations

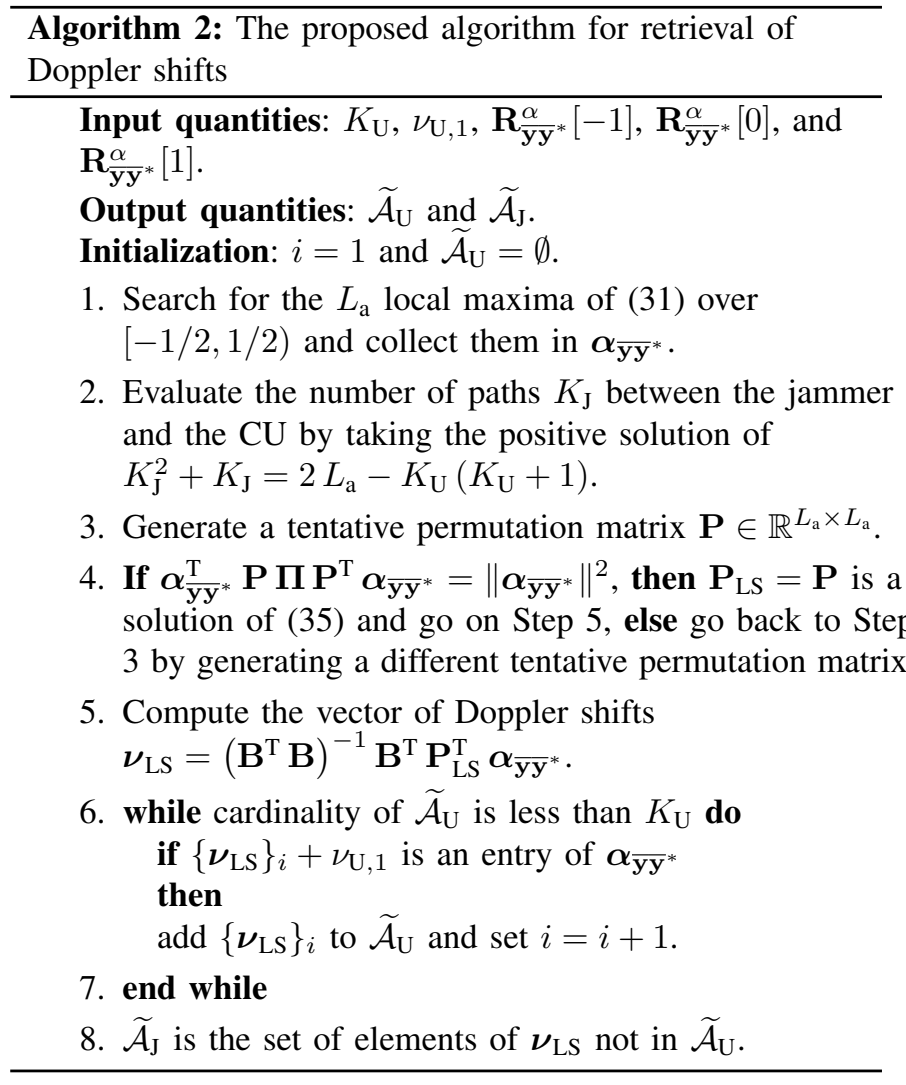

of the desired vector $\boldsymbol{\nu}_{\text {ord }}$. This inherent permutation ambiguity has to be solved in order to correctly distinguish the Doppler shifts of the UAV-to-CU transmission from those of the jammer-to-CU one. Remarkably, it is not necessary to completely remove such an ambiguity for detection purposes by singling $\boldsymbol{\nu}_{\text {ord }}$ out in $\left\{\boldsymbol{\nu}_{\mathrm{LS}, q}\right\}_{q=0}^{Q-1}$, but it is instead sufficient to separate the common entries of the vectors $\left\{\boldsymbol{\nu}_{\mathrm{LS}, q}\right\}_{q=0}^{Q-1}$ in two sets $\widetilde{\mathcal{A}}_{\mathrm{U}}$ and $\widetilde{\mathcal{A}}_{\mathrm{J}}$ : the former one gathers all the Doppler shifts of the UAV-to-CU transmission in any order ( $\mathcal{\mathcal { A }}_{\mathrm{U}}$ is the unordered version of $\mathcal{A}_{\mathrm{U}}$ ); the latter one collects all the Doppler shifts of the jammer-to-CU transmission in any $\operatorname{order}\left(\widetilde{\mathcal{A}}_{\mathrm{J}}\right.$ is the unordered version of $\left.\mathcal{A}_{\mathrm{J}}\right)$. This classification problem can be solved by resorting to Algorithm 2 reported at the top of this page, which is based on the additional knowledge of the Doppler shift $f_{\mathrm{U}, 1}$ (or, equivalently, $\nu_{\mathrm{U}, 1}$ ) of the LoS path between the UAV and the CU. ${ }^{7}$ The Doppler shift of such a LoS component depends on the relative speed, the transmitting wavelength, and the moving direction of the UAV with respect to the CU. Such parameters are commonly measured and/or estimated by the UAV relying on a set of sensors in conjunction with appropriate on-board algorithms and they are typically communicated to the $\mathrm{CU}$ through a

\footnotetext{
${ }^{7}$ Since floating point calculations are carried out with a finite precision depending on the used compilers and CPU architectures, the comparison between $\left\{\boldsymbol{\nu}_{\mathrm{LS}}\right\}_{i}+\nu_{\mathrm{U}, 1}$ and the $\ell$ th entry $\left\{\boldsymbol{\alpha}_{\overline{\mathbf{y y}}} *\right\}_{\ell}$ of $\boldsymbol{\alpha}_{\overline{\mathbf{y y}}} *$ in Algorithm 2 has to be numerically done by checking if the relative error $\left|\left\{\boldsymbol{\nu}_{\mathrm{LS}}\right\}_{i}+\nu_{\mathrm{U}, 1}-\left\{\boldsymbol{\alpha}_{\overline{\mathbf{y}}} *\right\}_{\ell}\right| /\left|\left\{\boldsymbol{\alpha}_{\overline{\mathbf{y}}} *\right\}_{\ell}\right|$ is smaller than a sufficiently small threshold $\epsilon>0$.
} 


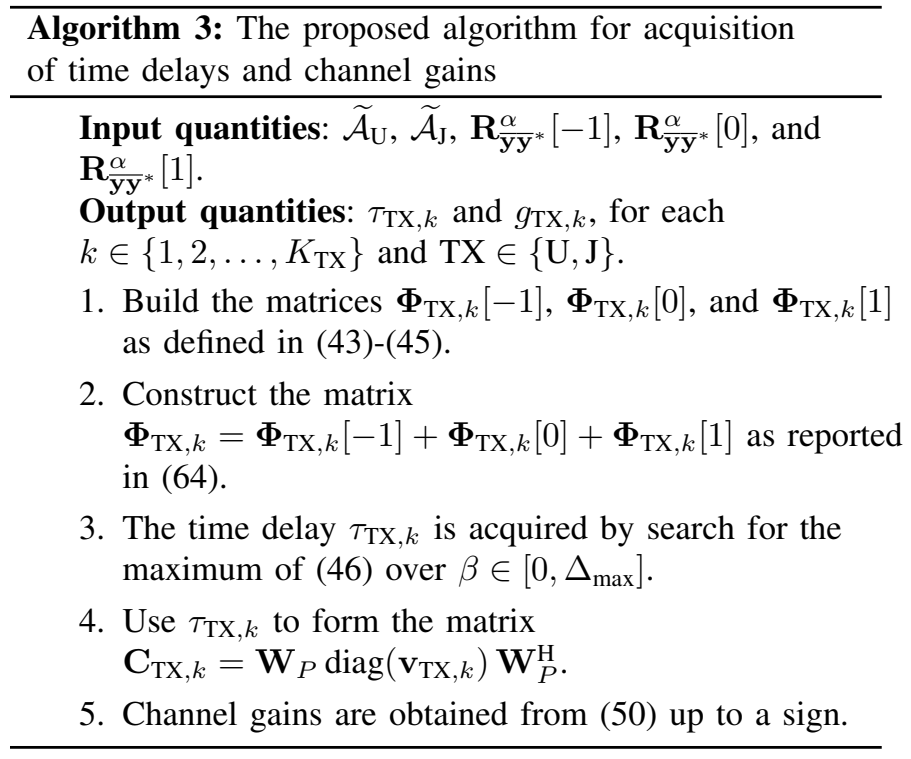

secure control and non-payload communication link [59].

A key observation underlying our blind estimation approach is that, in practice, $\mathbf{R}_{\mathbf{y y}^{*}}^{\alpha}[r]$ can be directly estimated from the received data using the consistent estimate (see, e.g., [60])

$$
\widehat{\mathbf{R}}_{\mathbf{y}^{*}}^{\alpha}[r]=\frac{1}{2 N+1} \sum_{n=-N}^{N} \overline{\mathbf{y}}[n] \overline{\mathbf{y}}^{\mathrm{T}}[n-r] e^{-j 2 \pi \alpha n}
$$

for $r \in\{-1,0,1\}$. Hence, in practice, an estimate $\widehat{\boldsymbol{\alpha}}_{\overline{\mathbf{y y}}^{*}}$ of the vector $\boldsymbol{\alpha}_{\overline{\mathbf{y y}}^{*}}$ can be directly obtained from data by finding the $\widehat{L}_{\mathrm{a}}$ distinct peaks of the function

$$
\begin{array}{r}
\widehat{J}(\alpha) \triangleq \sum_{r=-1}^{1}\left\|\widehat{\mathbf{R}}_{\overline{\mathbf{y y}}^{*}}^{\alpha}[r]\right\|^{2} \\
=\sum_{r=-1}^{1} \operatorname{tr}\left(\widehat{\mathbf{R}}_{\overline{\mathbf{y}}^{*}}^{\alpha}[r]\left\{\widehat{\mathbf{R}}_{\overline{\mathbf{y}}^{*}}^{\alpha}[r]\right\}^{\mathrm{H}}\right) \\
\text { with } \alpha \in[-1 / 2,1 / 2)
\end{array}
$$

which is the starting point to build the estimates $\widehat{\mathcal{A}}_{\mathrm{U}}$ and $\widehat{\mathcal{A}}_{\mathrm{J}}$ of the Doppler shift sets $\widetilde{\mathcal{A}}_{\mathrm{J}}$ and $\widetilde{\mathcal{A}}_{\mathrm{J}}$, respectively. The estimation procedure is a straightforward modification of Algorithm 2, with $J(\alpha), L_{\mathrm{a}}, \boldsymbol{\alpha}_{\overline{\mathbf{y y}^{*}}}$, and $\boldsymbol{\nu}_{\mathrm{LS}}$ replaced with $\widehat{J}(\alpha), \widehat{L}_{\mathrm{a}}$, $\widehat{\boldsymbol{\alpha}}_{\overline{\mathbf{y y}}{ }^{*}}$, and $\widehat{\boldsymbol{\nu}}_{\mathrm{LS}}=\left(\mathbf{B}^{\mathrm{T}} \mathbf{B}\right)^{-1} \mathbf{B}^{\mathrm{T}} \mathbf{P}_{\mathrm{LS}}^{\mathrm{T}} \widehat{\boldsymbol{\alpha}}_{\overline{\mathbf{y y}}^{*}}$, respectively, and the comparison between $\left\{\widehat{\boldsymbol{\nu}}_{\mathrm{LS}}\right\}_{i}+\nu_{\mathrm{U}, 1}$ and the $\ell$ th entry $\left\{\widehat{\boldsymbol{\alpha}}_{\overline{\mathbf{y y}}}\right\}_{\ell}$ of $\widehat{\boldsymbol{\alpha}}_{\overline{\mathbf{y y}}} *$ is performed by checking the corresponding relative error (see footnote 7 ). The consistency and asymptotic normality of estimate for $\widehat{J}(\alpha)$ can be proven by paralleling the derivations detailed in [40].

\section{Joint estimation of channel gains and time delays}

Hereinafter, we assume that the sets $\widetilde{\mathcal{A}}_{\mathrm{U}}$ and $\widetilde{\mathcal{A}}_{\mathrm{J}}$ are perfectly known. The proposed technique to jointly estimate the channel gains $g_{\mathrm{TX}, k}$ and delays $\tau_{\mathrm{TX}, k}=d_{\mathrm{TX}, k} T_{\mathrm{c}}+\chi_{\mathrm{TX}, k}$, for any $k \in\left\{1,2, \ldots, K_{\mathrm{TX}}\right\}$ and $\mathrm{TX} \in\{\mathrm{U}, \mathrm{J}\}$, also relies on the AP conjugate correlation matrices $\mathbf{R}_{\overline{\overline{y y}^{*}}}[n,-1], \mathbf{R}_{\overline{\mathbf{y y}}}{ }^{*}[n, 0]$, and $\mathbf{R}_{\overline{y_{\mathbf{y}}^{*}}}[n, 1]$ in (22). Specifically, let us consider the CCCMs (29), for $r \in\{-1,0,1\}$, at cycle frequency $\alpha=$ $\alpha_{\mathrm{TX}^{*}, k, k}=2 \nu_{\mathrm{TX}, k}$, which, according to (23)-(25), assume the forms reported in (40)-(42) at the top of the next page, for $k \in\left\{1,2, \ldots, K_{\mathrm{TX}}\right\}$ and $\mathrm{TX} \in\{\mathrm{U}, \mathrm{J}\}$. Relying on (40)-(42) and the previously acquired knowledge of the Doppler shifts, let us build the matrices

$$
\begin{aligned}
& \boldsymbol{\Phi}_{\mathrm{TX}, k}[-1] \triangleq e^{-j 2 \pi \nu_{\mathrm{TX}, k}} \mathbf{D}_{\mathrm{TX}, k}^{*} \mathbf{R}_{\overline{\mathbf{y}}^{*} \nu^{*}, k}^{2 \nu_{\mathrm{T} X}}[-1] \mathbf{D}_{\mathrm{TX}, k}^{*} \\
& =g_{\mathrm{TX}, k}^{2} \mathbf{T}_{\mathrm{TX}, k, 0} \boldsymbol{\Omega} \boldsymbol{\Omega}^{\mathrm{T}} \mathbf{T}_{\mathrm{TX}, k, 1}^{\mathrm{T}} \\
& \mathbf{\Phi}_{\mathrm{TX}, k}[0] \triangleq \mathbf{D}_{\mathrm{TX}, k}^{*} \mathbf{R}_{\overline{\mathbf{y y}}^{*} \nu_{\mathrm{TX}, k}}^{20]} \mathbf{D}_{\mathrm{TX}, k}^{*} \\
& =g_{\mathrm{TX}, k}^{2}\left(\mathbf{T}_{\mathrm{TX}, k, 0} \boldsymbol{\Omega} \boldsymbol{\Omega}^{\mathrm{T}} \mathbf{T}_{\mathrm{TX}, k, 0}^{\mathrm{T}}\right. \\
& \left.+\mathbf{T}_{\mathrm{TX}, k, 1} \boldsymbol{\Omega} \boldsymbol{\Omega}^{\mathrm{T}} \mathbf{T}_{\mathrm{TX}, k, 1}^{\mathrm{T}}\right) \\
& \boldsymbol{\Phi}_{\mathrm{TX}, k}[1] \triangleq e^{j 2 \pi \nu_{\mathrm{TX}, k}} \mathbf{D}_{\mathrm{TX}, k}^{*} \mathbf{R}_{\overline{\mathbf{y}} \mathbf{y}^{*}}^{2 \nu_{\mathrm{TX}, k}}[1] \mathbf{D}_{\mathrm{TX}, k}^{*} \\
& =g_{\mathrm{TX}, k}^{2} \mathbf{T}_{\mathrm{TX}, k, 1} \boldsymbol{\Omega} \boldsymbol{\Omega}^{\mathrm{T}} \mathbf{T}_{\mathrm{TX}, k, 0}^{\mathrm{T}} .
\end{aligned}
$$

Proposition 4.2: Acquisition of the delay $\tau_{\mathrm{TX}, k}$ can be pursued by searching over the interval $\left[0, \Delta_{\max }\right] \subset[0, T / 2)$ for the global maximum of the one-dimensional cost function defined in (46) at the top of the next page, where

$$
\begin{aligned}
\boldsymbol{\Phi}_{\mathrm{TX}, k} & \triangleq \sum_{r=-1}^{1} \boldsymbol{\Phi}_{\mathrm{TX}, k}[r] \\
\mathbf{\Upsilon} & \triangleq \mathbf{W}_{P}^{\mathrm{H}} \boldsymbol{\Omega} \boldsymbol{\Omega}^{\mathrm{T}} \mathbf{W}_{P}^{*} \in \mathbb{C}^{P \times P}
\end{aligned}
$$

and, for $p \in\{0,1, \ldots, P-1\}$, the sample $\Psi_{\mathrm{TX}, k}[p]$ is given by (74)-(75) in Appendix C.

Proof: See Appendix B.

Once the Doppler shifts and delays are acquired, the fading coefficients can be estimated from (64) by solving the LS optimization problem:

$$
\arg \min _{\rho \in \mathbb{C}}\left\|\mathbf{\Phi}_{\mathrm{TX}, k}-\rho^{2} \mathbf{C}_{\mathrm{TX}, k} \boldsymbol{\Omega} \boldsymbol{\Omega}^{\mathrm{T}} \mathbf{C}_{\mathrm{TX}, k}^{\mathrm{T}}\right\|^{2}
$$

from which one gets

$$
g_{\mathrm{TX}, k}^{2}=\frac{\operatorname{tr}\left(\mathbf{\Phi}_{\mathrm{TX}, k} \mathbf{C}_{\mathrm{TX}, k}^{*} \boldsymbol{\Omega}^{*} \boldsymbol{\Omega}^{\mathrm{H}} \mathbf{C}_{\mathrm{TX}, k}^{\mathrm{H}}\right)}{\operatorname{tr}\left(\mathbf{C}_{\mathrm{TX}, k} \boldsymbol{\Omega} \boldsymbol{\Omega}^{\mathrm{T}} \mathbf{C}_{\mathrm{TX}, k}^{\mathrm{T}} \mathbf{C}_{\mathrm{TX}, k}^{*} \boldsymbol{\Omega}^{*} \boldsymbol{\Omega}^{\mathrm{H}} \mathbf{C}_{\mathrm{TX}, k}^{\mathrm{H}}\right)}
$$

for $k \in\left\{1,2, \ldots, K_{\mathrm{TX}}\right\}$ and $\mathrm{TX} \in\{\mathrm{U}, \mathrm{J}\}$. Since (50) provides the square of the complex gain $g_{\mathrm{TX}, k}$, there is a $\pi$-radian phase ambiguity associated with the recovery of $\measuredangle g_{\mathrm{TX}, k}$. Such a sign ambiguity can be removed by building all the $2^{K_{\mathrm{U}}+K_{\mathrm{J}}}$ possible matrices $\widetilde{\mathbf{H}}[n]$ in (11) and, then, choosing the one that yields the best pre-detection performance. The whole estimation process is summarized in Algorithm 3 reported at the top of this page.

In practice, an estimate $\widehat{\tau}_{\mathrm{TX}, k}$ of the delay $\tau_{\mathrm{TX}, k}$ is derived by searching for the peak of the finite-sample version $\widehat{\mathcal{I}}_{\mathrm{TX}, k}(\beta)$ of the cost function $\mathcal{I}_{\mathrm{TX}, k}(\beta)$ defined in (46), where $\widehat{\mathcal{I}}_{\mathrm{TX}, k}(\beta)$ is obtained by replacing $\mathbf{R}_{\overline{\mathbf{y y}}^{*}}^{2 \nu_{\mathrm{TX}, k}}[r]$ in (43)-(45), for $r \in\{-1,0,1\}$, with their corresponding estimates (38), evaluated at the estimated cycle frequency $\alpha=2 \widehat{\nu}_{\mathrm{TX}, k}$. Additionally, the estimate $\widehat{\nu}_{\mathrm{TX}, k}$ of the $k$ th Doppler shift associated with the TX-to-CU channel - which has been 


$$
\begin{aligned}
& \mathbf{R}_{\mathbf{y y}^{*}}^{2 \nu_{\text {TX }, k}}[-1]=g_{\mathrm{TX}, k}^{2} e^{j 2 \pi \nu_{\mathrm{TX}, k}} \mathbf{D}_{\mathrm{TX}, k} \mathbf{T}_{\mathrm{TX}, k, 0} \boldsymbol{\Omega} \boldsymbol{\Omega}^{\mathrm{T}} \mathbf{T}_{\mathrm{TX}, k, 1}^{\mathrm{T}} \mathbf{D}_{\mathrm{TX}, k} \\
& \mathbf{R}_{\overline{\mathbf{y}} \mathbf{y}^{*}{ }^{2}, k}^{2}[0]=g_{\mathrm{TX}, k}^{2} \mathbf{D}_{\mathrm{TX}, k}\left(\mathbf{T}_{\mathrm{TX}, k, 0} \boldsymbol{\Omega} \boldsymbol{\Omega}^{\mathrm{T}} \mathbf{T}_{\mathrm{TX}, k, 0}^{\mathrm{T}}+\mathbf{T}_{\mathrm{TX}, k, 1} \boldsymbol{\Omega} \boldsymbol{\Omega}^{\mathrm{T}} \mathbf{T}_{\mathrm{TX}, k, 1}^{\mathrm{T}}\right) \mathbf{D}_{\mathrm{TX}, k} \\
& \mathbf{R}_{\overline{\mathbf{y y}^{*}}}^{2 \nu_{\mathrm{TX}, k}}[1]=g_{\mathrm{TX}, k}^{2} e^{-j 2 \pi \nu_{\mathrm{TX}, h}} \mathbf{D}_{\mathrm{TX}, k} \mathbf{T}_{\mathrm{TX}, k, 1} \boldsymbol{\Omega} \boldsymbol{\Omega}^{\mathrm{T}} \mathbf{T}_{\mathrm{TX}, k, 0}^{\mathrm{T}} \mathbf{D}_{\mathrm{TX}, k}
\end{aligned}
$$

$$
\begin{aligned}
\mathcal{I}_{\mathrm{TX}, k}(\beta) & \triangleq\left|\sum_{p=0}^{P / 2-1}\left\{\mathbf{W}_{P}^{\mathrm{H}} \mathbf{\Phi}_{\mathrm{TX}, k} \mathbf{W}_{P}^{*}\right\}_{p, p}\left(\Psi_{\mathrm{TX}, k}^{2}[p]\right)^{*}\{\boldsymbol{\Upsilon}\}_{p, p}^{*} e^{j \frac{4 \pi}{T} \beta p}\right| \\
& =\left.\left|g_{\mathrm{TX}, k}\right|^{2}\left|\sum_{p=0}^{P / 2-1}\right| \Psi_{\mathrm{TX}, k}[p]\right|^{4}\left|\{\boldsymbol{\Upsilon}\}_{p, p}\right|^{2} e^{-j \frac{4 \pi}{T}\left(\tau_{\mathrm{TX}, k}-\beta\right) p} \mid, \quad \text { with } \beta \in\left[0, \Delta_{\max }\right]
\end{aligned}
$$

TABLE III

MSE OF UAV CHANNEL PARAMETERS (HIGH-SPEED JAMMER).

\begin{tabular}{cccc}
\hline MSE $(\mathrm{dB})$ & $\mathrm{SJR}=-3 \mathrm{~dB}$ & $\mathrm{SJR}=0 \mathrm{~dB}$ & $\mathrm{SJR}=3 \mathrm{~dB}$ \\
\hline \hline Channel gain & -33.4944 & -37.1155 & -39.7152 \\
\hline Doppler shift & -63.8131 & -63.8131 & -63.8859 \\
\hline Time delay & -39.7772 & -43.4395 & -46.1916 \\
\hline \hline
\end{tabular}

TABLE IV

MSE OF JAMMER CHANNEL PARAMETERS (HIGH-SPEED JAMMER).

\begin{tabular}{cccc}
\hline MSE $(\mathrm{dB})$ & $\mathrm{SJR}=-3 \mathrm{~dB}$ & $\mathrm{SJR}=0 \mathrm{~dB}$ & $\mathrm{SJR}=3 \mathrm{~dB}$ \\
\hline \hline Channel gain & -41.5433 & -38.4094 & -34.2107 \\
\hline Doppler shift & -70.0077 & -70.0077 & -69.9169 \\
\hline Time delay & -46.6658 & -43.1931 & -38.3779 \\
\hline \hline
\end{tabular}

previously achieved as explained in Subsection IV-C - has to be used to replace $\mathbf{D}_{\mathrm{TX}, k}$ in (43)-(45) with its estimate $\widehat{\mathbf{D}}_{\mathrm{TX}, k} \triangleq \operatorname{diag}\left(1, e^{j \frac{2 \pi}{P} \widehat{\nu}_{\mathrm{TX}, k}}, \ldots, e^{j \frac{2 \pi}{P} \widehat{\nu}_{\mathrm{TX}, k}(P-1)}\right)$. So doing, one constructs the matrices

$$
\begin{aligned}
& \widehat{\boldsymbol{\Phi}}_{\mathrm{TX}, k}[-1]=e^{-j 2 \pi \widehat{\nu}_{\mathrm{TX}, k}} \widehat{\mathbf{D}}_{\mathrm{TX}, k}^{*} \widehat{\mathbf{R}}_{\overline{\mathbf{Y y}}^{*}}^{2 \widehat{\nu}_{\mathrm{TX}, k}}[-1] \widehat{\mathbf{D}}_{\mathrm{TX}, k}^{*} \\
& \widehat{\boldsymbol{\Phi}}_{\mathrm{TX}, k}[0]=\widehat{\mathbf{D}}_{\mathrm{TX}, k}^{*} \widehat{\mathbf{R}}_{\overline{\mathbf{y y}}^{*}}^{2 \widehat{\nu}_{\mathrm{XX}, k}}[0] \widehat{\mathbf{D}}_{\mathrm{TX}, k}^{*} \\
& \widehat{\boldsymbol{\Phi}}_{\mathrm{TX}, k}[1]=e^{j 2 \pi \widehat{\nu}_{\mathrm{TX}, k}} \widehat{\mathbf{D}}_{\mathrm{TX}, k}^{*} \widehat{\mathbf{R}}_{\overline{\mathbf{y}}^{*}}^{2 \widehat{\nu}_{\mathrm{TX}, k}}[1] \widehat{\mathbf{D}}_{\mathrm{TX}, k}^{*} \\
& \widehat{\boldsymbol{\Phi}}_{\mathrm{TX}, k}=\widehat{\boldsymbol{\Phi}}_{\mathrm{TX}, k}[-1]+\widehat{\boldsymbol{\Phi}}_{\mathrm{TX}, k}[0]+\widehat{\boldsymbol{\Phi}}_{\mathrm{TX}, k}[1] .
\end{aligned}
$$

After calculating $\widehat{\tau}_{\mathrm{TX}, k}$, the corresponding estimate $\widehat{g}_{\mathrm{TX}, k}$ of the channel gain $g_{\mathrm{TX}, k}$ is obtained from (50) by replacing $\boldsymbol{\Phi}_{\mathrm{TX}, k}$ and $\mathbf{C}_{\mathrm{TX}, k}$ with their corresponding estimates $\widehat{\boldsymbol{\Phi}}_{\mathrm{TX}, k}$ and $\widehat{\mathbf{C}}_{\mathrm{TX}, k}=\mathbf{W}_{P} \operatorname{diag}\left(\widehat{\mathbf{v}}_{\mathrm{TX}, k}\right) \mathbf{W}_{P}^{\mathrm{H}}$, respectively, where the $p$ th entry of $\widehat{\mathbf{v}}_{\mathrm{TX}, k}$ is given by $\left\{\widehat{\mathbf{v}}_{\mathrm{TX}, k}\right\}_{p}=\widehat{\Psi}_{\mathrm{TX}, k}[p] e^{-j \frac{2 \pi}{T} \widehat{T}_{\mathrm{TX}, k} p}$, for $p \in\{0,1, \ldots, P-1\}$, and $\widehat{\Psi}_{\mathrm{TX}, k}[p]$ is obtained from (75) in the Appendix by replacing $\chi_{\mathrm{TX}, k}$ with $\hat{\chi}_{\mathrm{TX}, k}$.

Study of the asymptotic behavior of the proposed estimators of channel gains and time delays is a challenging mathematical problem. The performances of these estimators are assessed by simulations in Section V.
TABLE V

MSE OF UAV CHANNEL PARAMETERS (LOW-SPEED JAMMER).

\begin{tabular}{cccc}
\hline MSE $(\mathrm{dB})$ & $\mathrm{SJR}=-3 \mathrm{~dB}$ & $\mathrm{SJR}=0 \mathrm{~dB}$ & $\mathrm{SJR}=3 \mathrm{~dB}$ \\
\hline \hline Channel gain & -35.4503 & -39.4990 & -42.3258 \\
\hline Doppler shift & -63.4707 & -63.4707 & -63.4707 \\
\hline Time delay & -38.7822 & -43.5352 & -46.5625 \\
\hline \hline
\end{tabular}

TABLE VI

MSE OF JAMMER CHANNEL PARAMETERS (LOW-SPEED JAMMER).

\begin{tabular}{cccc}
\hline MSE $(\mathrm{dB})$ & $\mathrm{SJR}=-3 \mathrm{~dB}$ & $\mathrm{SJR}=0 \mathrm{~dB}$ & $\mathrm{SJR}=3 \mathrm{~dB}$ \\
\hline \hline Channel gain & -41.9432 & -38.8324 & -34.4111 \\
\hline Doppler shift & -57.1067 & -57.2068 & -57.2068 \\
\hline Time delay & -45.9246 & -43.2448 & -39.4336 \\
\hline \hline
\end{tabular}

\section{NUMERICAL RESULTS}

In this Section, we provide simulation results aimed at evaluating the performance of the considered anti-jamming detector (see Algorithm 1), equipped with the proposed channel estimators (see Algorithms 2 and 3). To this aim, we consider the following simulation setting. The UAV and the mobile jammer employ OFDM modulation with $M=16$ subcarriers, $\mathrm{CP}$ of length $L_{\mathrm{cp}}=4$, sampling rate $1 / T_{\mathrm{c}}=625$ $\mathrm{kHz}$, and binary-phase-shift-keying (BPSK) signaling. The carrier frequency is set to $f_{0}=27 \mathrm{GHz}$. The number of paths of the UAV-to-CU and jammer-to-CU links are fixed to $K_{\mathrm{U}}=K_{\mathrm{J}}=2$. For $k \in\{1,2\}$ and $\mathrm{TX} \in\{\mathrm{U}, \mathrm{J}\}$, the gains $g_{\mathrm{TX}, k}$ of both the UAV and jammer channels are generated as statistically independent Rayleigh random variables (RVs), with

$$
\sigma_{\mathrm{TX}}^{2} \triangleq \mathbb{E}\left(\left|g_{\mathrm{TX}, k}\right|^{2}\right)=2 \mathcal{P}_{\mathrm{TX}}\left(\frac{\lambda_{0}}{4 \pi d_{\mathrm{TX}}}\right)^{2}
$$

where $\lambda_{0}=c_{0} / f_{0}$ is the wavelength, $c_{0}=3 \cdot 10^{8} \mathrm{~m} / \mathrm{s}$ denotes the light speed, and $d_{\mathrm{TX}}$ is the distance between TX and the $\mathrm{CU}$, whereas the delays $\tau_{\mathrm{TX}, k}$ are randomly generated according to the one-sided exponentially decreasing delay 

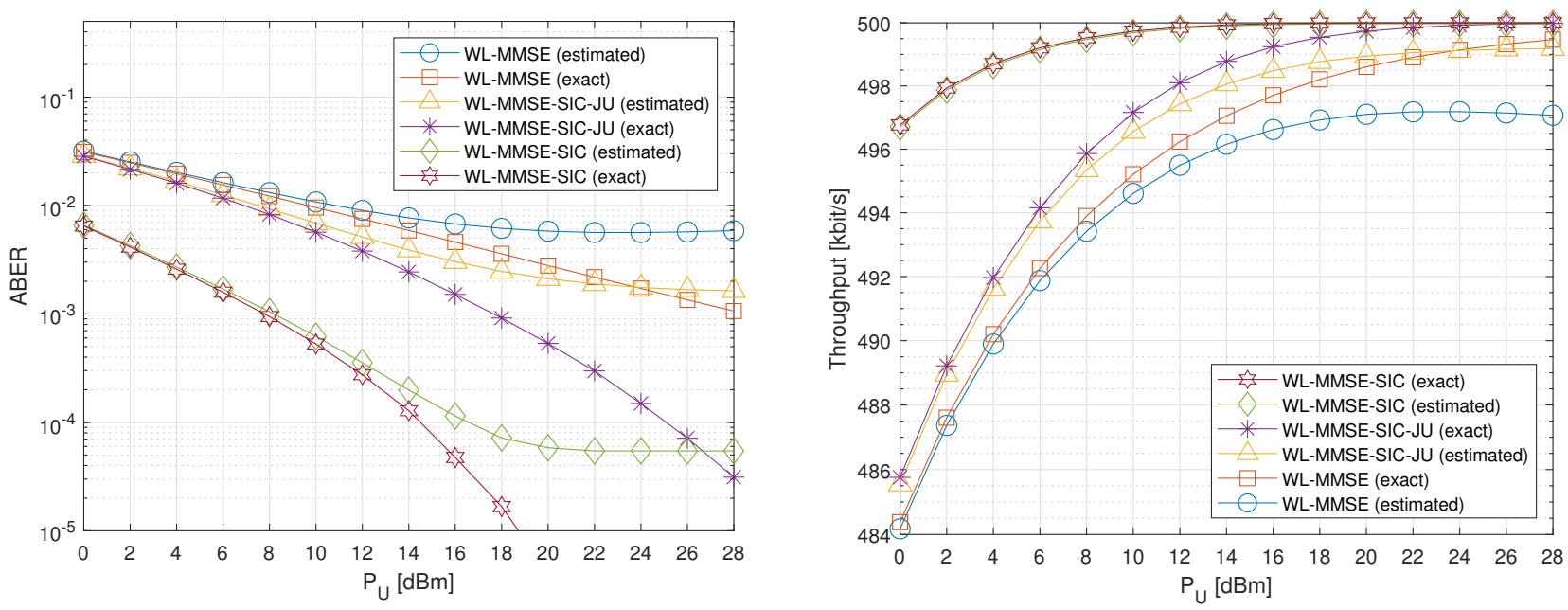

Fig. 3. ABER (left) and throughput (right) versus $\mathrm{SNR}$ ( $\mathrm{SJR}=-3 \mathrm{~dB}$, high-speed jammer).
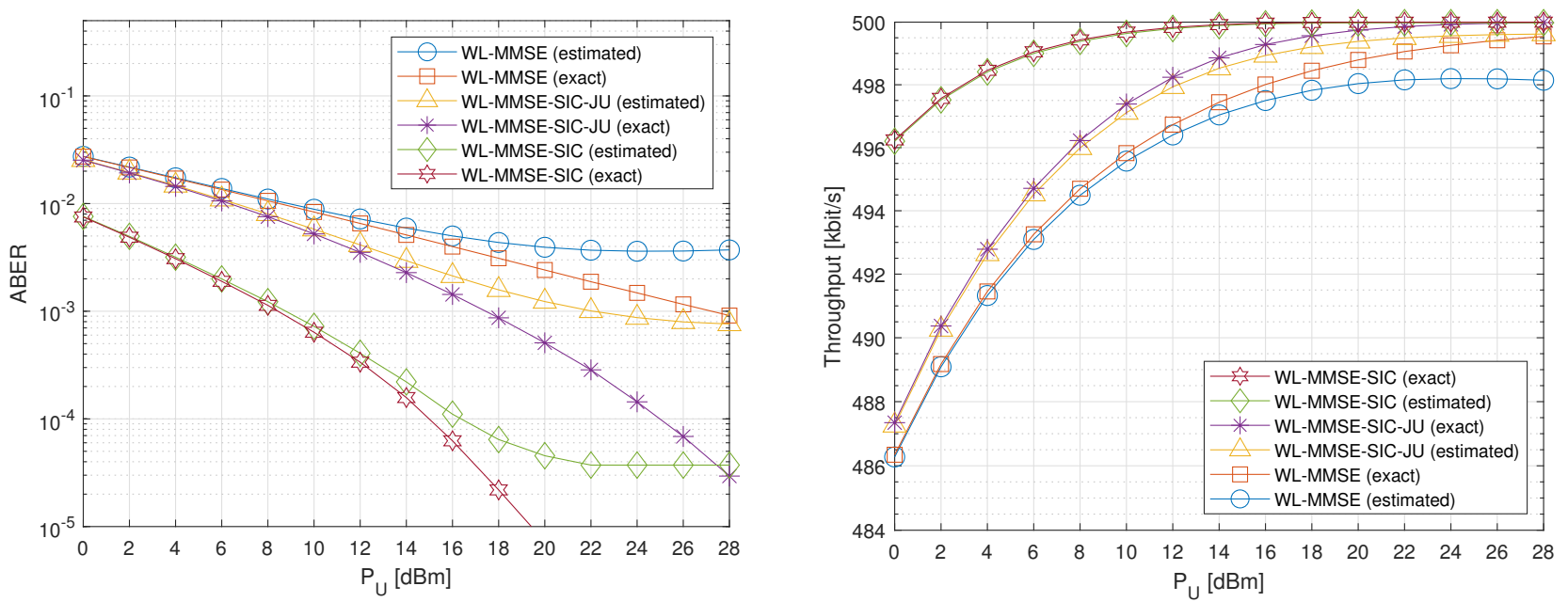

Fig. 4. ABER (left) and throughput (right) versus SNR ( $\mathrm{SJR}=0 \mathrm{~dB}$, high-speed jammer).
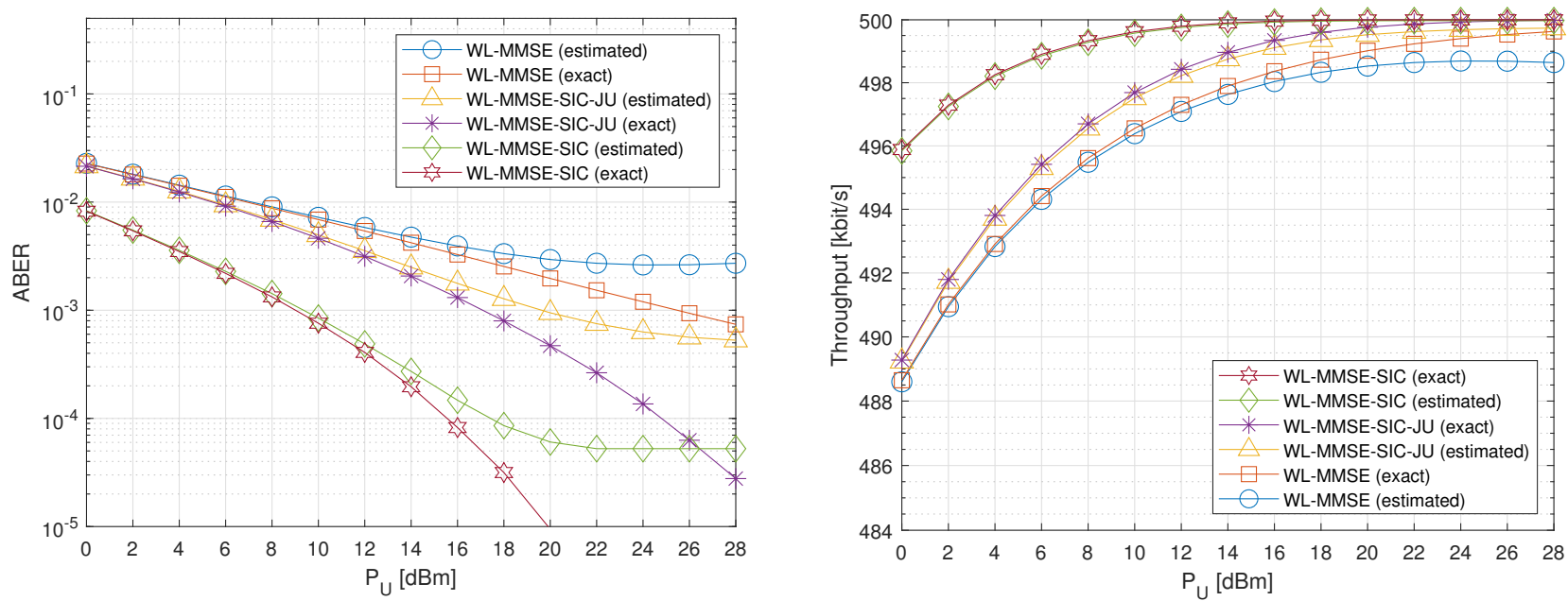

Fig. 5. ABER (left) and throughput (right) versus $\mathrm{SNR}$ ( $\mathrm{SJR}=3 \mathrm{~dB}$, high-speed jammer). 

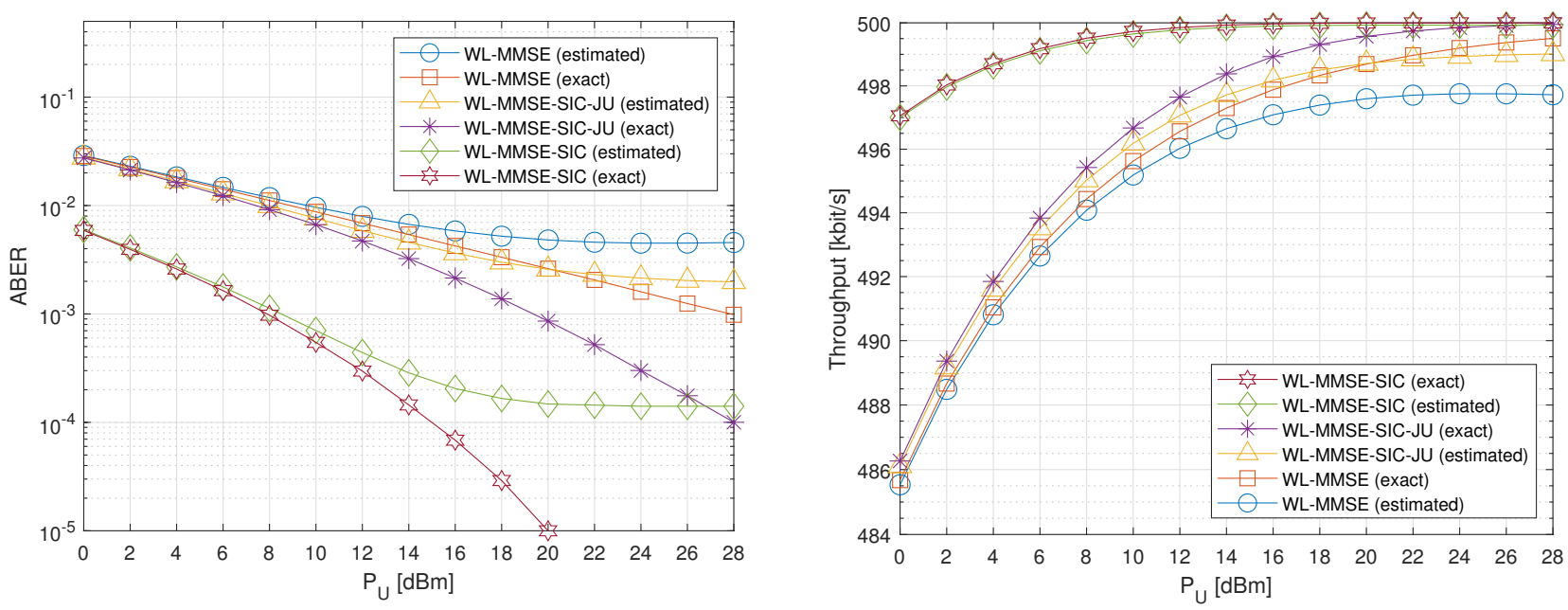

Fig. 6. ABER (left) and throughput (right) versus SNR (SJR $=-3 \mathrm{~dB}$, low-speed jammer).
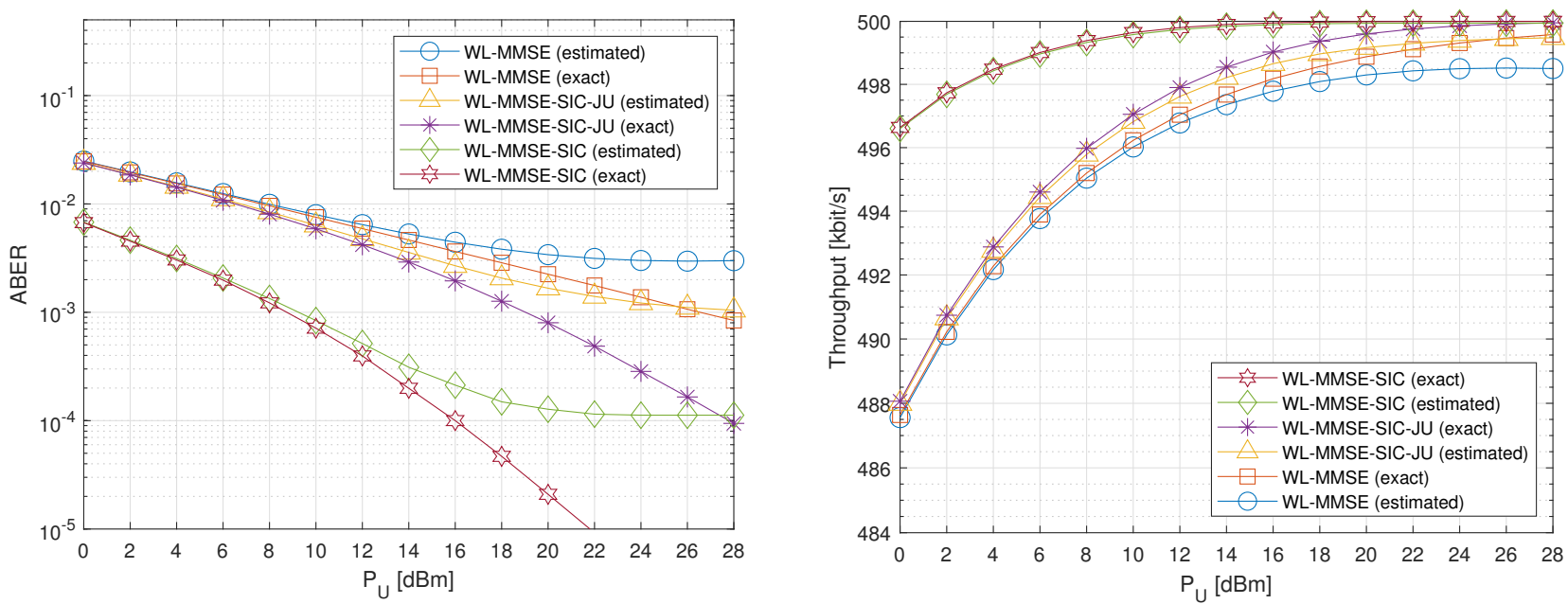

Fig. 7. ABER (left) and throughput (right) versus SNR (SJR $=0 \mathrm{~dB}$, low-speed jammer).
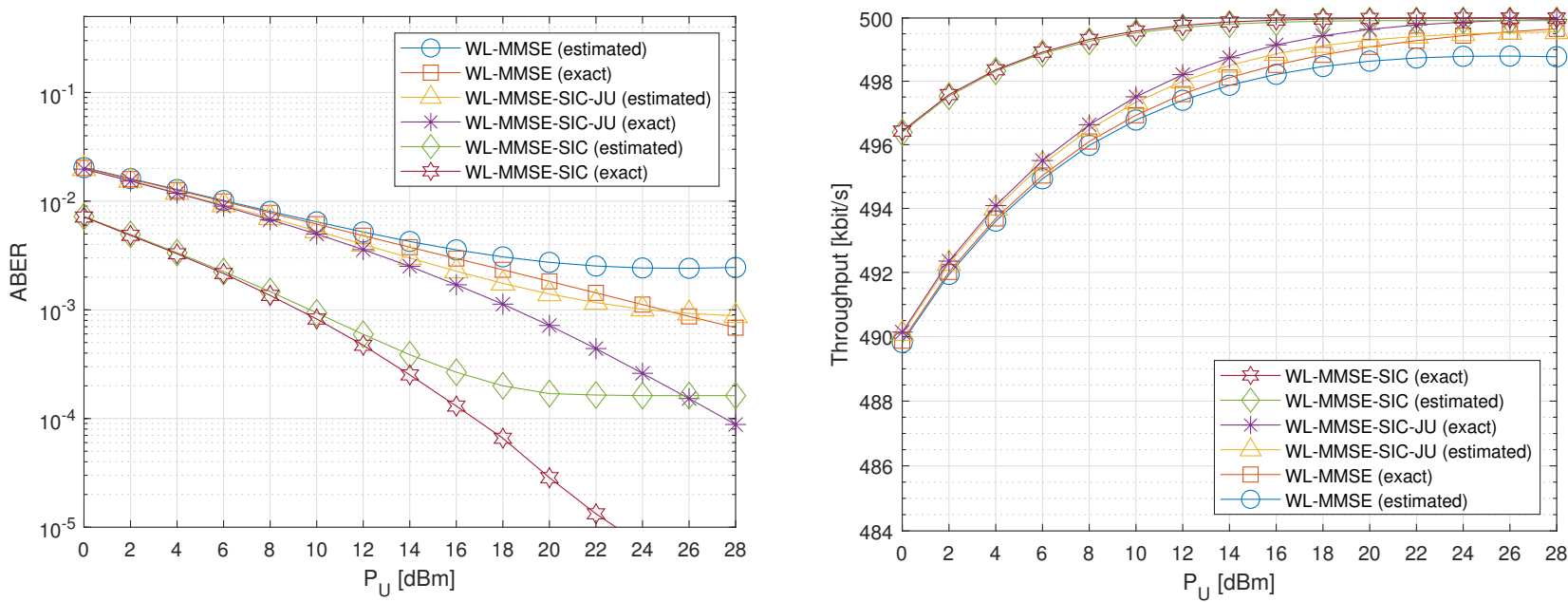

Fig. 8. ABER (left) and throughput (right) versus SNR ( $\mathrm{SJR}=3 \mathrm{~dB}$, low-speed jammer). 
power spectrum [64], i.e.,

$$
\tau_{\mathrm{TX}, k}=-\tau_{\text {slope }} \ln \left[1-u_{k}\left(1-e^{-\Delta_{\mathrm{TX}} / \tau_{\text {slope }}}\right)\right]
$$

where the maximum delay is $\Delta_{\mathrm{U}}=\Delta_{\mathrm{J}}=3 T_{\mathrm{c}}$, the slopetime is $\tau_{\text {slop }}=2 T_{\mathrm{c}}$, and $u_{k}$ are independent RVs uniformly distributed in $[0,1]$. We set $d_{\mathrm{U}}=d_{\mathrm{J}}=100 \mathrm{~m}$ and the noise variance $\sigma_{w}^{2}$ is equal to $-113 \mathrm{dBm}$.

We assume that the flight speed of the UAV is $v_{\mathrm{U}}=10 \mathrm{~m} / \mathrm{s}$, while we consider two mobility scenarios for the jammer:

1) high-speed jammer: the jammer moves at $v_{\mathrm{J}}=20 \mathrm{~m} / \mathrm{s}$;

2) low-speed jammer: the jammer moves at $v_{\mathrm{J}}=5 \mathrm{~m} / \mathrm{s}$.

The Doppler frequencies $f_{\mathrm{TX}, k}$ are customarily generated as $f_{\mathrm{TX}, k}=D_{\mathrm{TX}} \cos \left(\vartheta_{\mathrm{TX}, k}\right)$, where $D_{\mathrm{TX}} \triangleq\left(v_{\mathrm{TX}} / c_{0}\right) f_{0}$ and $\vartheta_{\mathrm{TX}, k}$ are independent RVs, uniformly distributed in $[0,2 \pi)$. During the downloading phase, the UAV transmits $5 \mathrm{MB}$ of collected data.

In each Monte Carlo run, a new set of symbols, noise and channel parameters for both the UAV and jammer are randomly generated. As a performance measure, we report the average bit error rate (ABER) at the $\mathrm{CU}$ regarding the UAV transmission and the corresponding throughput, ${ }^{8}$ as well as the arithmetic mean of the MSEs of the Doppler shifts $f_{\mathrm{TX}, k}$ (normalized by $D_{\mathrm{TX}}^{2}$ ), the time delays $\tau_{\mathrm{TX}, k}$ (normalized by $\Delta_{\mathrm{TX}}^{2}$ ), and channel gains $g_{\mathrm{TX}, k}$ (normalized by $\sigma_{\mathrm{TX}}^{2}$ ), for $k \in\{1,2\}$ and $\mathrm{TX} \in\{\mathrm{U}, \mathrm{J}\}$. Three different values of $\mathrm{SJR} \in\{-3,0,3\} \mathrm{dB}$ are considered, with $\mathrm{SJR} \triangleq \sigma_{\mathrm{U}}^{2} / \sigma_{\mathrm{J}}^{2}$, representing the situations in which the transmit power of the UAV is half, equal to, or double of that of the jammer, respectively.

In addition to the proposed WL symbol detection procedure with anti-jamming capabilities based on serial interference cancellation (SIC), we also evaluated the performance of the simpler WL-MMSE detector. We also implemented the WLMMSE-SIC jamming-unaware $(J U)$ detector, which recovers the UAV symbol vector $\mathbf{s}_{\mathrm{U}}[n]$ - rather than both $\mathbf{s}_{\mathrm{U}}[n]$ and $\mathbf{s}_{\mathrm{J}}[n]$ - and involves only the knowledge of $\mathbf{H}_{\mathrm{U}}[n]$, thereby treating the jammer as a disturbance. The ABER curves of all the considered detection techniques are reported in the case of perfect knowledge of $\mathbf{H}_{\mathrm{U}}[n]$ and $\mathbf{H}_{\mathbf{J}}[n]$, referred to as "exact", as well as when the channel state information is built by using the estimates of the relevant channel parameters (i.e., Doppler shifts, time delays, and channel gains) obtained from the received data, referred to as "estimated".

\section{A. Scenario 1: High-speed jammer}

The performance of the proposed finite-sample versions of the estimators outlined in Algorithms 2 and 3 is reported in Tables III and IV for the UAV and jammer transmissions, respectively, when $\mathcal{P}_{\mathrm{U}}=10 \mathrm{dBm}$ and $v_{\mathrm{J}}=20 \mathrm{~m} / \mathrm{s}$. It is apparent that the proposed estimators exhibit very satisfactory MSE performance for all the considered SJR values. Remarkably, the estimation accuracy of the Doppler shifts is

\footnotetext{
${ }^{8}$ For the considered simulation setting, the maximum achievable throughpu is given by $\left(1 / T_{\mathrm{c}}\right)(M / P)=625 \cdot 0.8=500 \mathrm{kbit} / \mathrm{s}$, due to the redundancy arising from the $\mathrm{CP}$ insertion at the OFDM transmitter.
}

almost insensitive to the SJR. It is also evident that, compared with the UAV case, the channel parameters of the jammer are estimated with a better accuracy. This is due to the fact that a larger value of the transmitter speed enables a better estimate of its cycle frequencies, which in its turn has a positive effect on the estimation process of the time delays and channel gains, whose estimators require prior acquisition of the Doppler shifts. Results not reported here show that the estimation accuracy is the same for different values of $\mathcal{P}_{U}$.

Figs. 3, 4, and 5 depict the ABER (left-side plot) and throughput (right-side plot) performance of the WL-MMSE detector and the proposed WL-MMSE-SIC one, whose synthesis is detailed in Algorithm 1, as well as the performance of the WL-MMSE-SIC-JU receiver. As confirmed by our theoretical prediction, the system performance when the WL-MMSE detector is employed at the $\mathrm{CU}$ is seriously degraded by the residual ICI and jamming contributions at its output. In particular, the data-estimated version of the WL-MMSE detector exhibits a high ABER floor well above $10^{-3}$, which in its turn leads to a corresponding throughput floor. Compared with the WL-MMSE detector, the WL-MMSE-SIC-JU receiver allows one to significantly improve the system performance in ideal conditions (i.e., exact knowledge of $\mathbf{H}_{\mathrm{U}}[n]$ and the correlation matrix of $\tilde{\mathbf{y}}_{i}$, for $i \in\{0,1, \ldots, 2 M-1\}$ ), but pays an acceptable performance degradation when implemented from data, due to the presence of a high-power jammer. On the other hand, the proposed WL-MMSE-SIC detector is able to effectively remove the residual ICI and jamming impairments after the WL-MMSE pre-detection, by ensuring performances that are almost unaffected by the considered SJR values in both the exact and estimated versions. Specifically, the dataestimated version of the WL-MMSE-SIC shows a BER floor only below $10^{-4}$, due to the finite-sample estimation of the relevant channel parameters, which does not prevent to achieve the maximum throughput.

\section{B. Scenario 2: Low-speed jammer}

The numerical experiment of the previous Section is repeated for the case of $v_{\mathrm{J}}=5 \mathrm{~m} / \mathrm{s}$ and the corresponding results are reported in Tables V and VI, as well as in Figs. 6, 7, and 8. It is seen that, as intuitively expected, the only noticeable effect of a lower speed of the jammer is a reduction of the estimation accuracy of its Doppler shifts, which however has a negligible impact on ABER and throughput performance of the UAV transmission.

\section{CONCLUSIONS AND DIRECTIONS FOR FUTURE WORK}

In this paper, we have investigated anti-jamming communications in UAV-aided WSNs operating over doubly-selective channels for smart city applications. We have focused on the downloading phase, when the UAV is subject to a jamming attack when it is transmitting to a remote CU the data collected through the city.

The following countermeasures have been proposed:

(i) Joint detection of the UAV and jammer symbols is performed to address the case of strong ICI and jamming 
power, in conjunction with serial cancellation of the residual ICI and jamming contribution based on postsorting of the detector output.

(ii) The Doppler shifts, time delays and channel gains required to implement the improved detection strategy are blindly acquired by exploiting the ACS properties of the received signals through algorithms that exploit the detailed structure of multicarrier modulation format.

In summary, this study demonstrates that, if sophisticated reception strategies and channel estimators are employed during jamming attack, it is still possible to ensure a satisfactory performance in terms of ABER, throughput, and MSE of the estimated channel parameters, which is only slightly affected by the transmitted power of the jammer. It is worth noting that the proposed countermeasures can be used outside the application scenario of smart cities, whenever a large amount of data is available and latency constraints are relaxed. Finally, we have studied the performance of the proposed estimators by means of numerical simulations. In this respect, a first interesting research subject consists of investigating the consistency and asymptotic distribution of the developed estimators from a theoretical viewpoint. To further corroborate the effectiveness of the proposed anti-jamming countermeasures, an additional research issue is to deploy a physical testbed by programming general purpose radio hardware according to the softwaredefined paradigm.

\section{APPENDIX A}

\section{PROOF OF PROPOSITION 4.1}

Lat us take into account two different linear mappings: the former one is given by

$$
\mathcal{L}_{1}: \boldsymbol{\nu}_{\text {ord }} \in[-1 / 2,1 / 2)^{K} \longrightarrow \boldsymbol{\alpha}_{\text {ord }} \in[-1 / 2,1 / 2)^{L_{\mathrm{a}}}
$$

where we remember that $\boldsymbol{\nu}_{\text {ord }}=\left(\boldsymbol{\nu}_{\mathrm{U}}^{\mathrm{T}}, \boldsymbol{\nu}_{\mathrm{J}}^{\mathrm{T}}\right)^{\mathrm{T}} \in[-1 / 2,1 / 2)^{K}$, with $K \triangleq K_{\mathrm{U}}+K_{\mathrm{J}}$, and

$$
\begin{aligned}
& \boldsymbol{\alpha}_{\text {ord }} \triangleq\left(\alpha_{\mathrm{U}^{(*)}, 1,1}, \ldots, \alpha_{\mathrm{U}^{(*)}, K_{\mathrm{U}}, K_{\mathrm{U}}}, \alpha_{\mathbf{J}(*), 1,1}, \ldots, \alpha_{\mathbf{J}(*)}, K_{\mathrm{J}}, K_{\mathrm{J}},\right. \\
& \alpha_{\mathrm{U}^{(*)}, 1,2}, \ldots, \alpha_{\mathrm{U}^{(*)}, 1, K_{\mathrm{U}}}, \alpha_{\mathbf{J}^{(*)}, 1,2}, \ldots, \alpha_{\mathbf{J}^{(*)}, 1, K_{\mathrm{J}}}, \\
& \alpha_{\mathrm{U}^{(*)}, 2,3}, \ldots, \alpha_{\mathrm{U}^{(*)}, 2, K_{\mathrm{U}}}, \alpha_{\mathbf{J}^{(*)}, 2,3}, \ldots, \alpha_{\mathbf{J}^{(*)}, 2, K_{\mathrm{U}}}, \ldots \\
& \left.\alpha_{\mathrm{U}^{(*)}, K_{\mathrm{U}}-1, K_{\mathrm{U}}}, \alpha_{\mathbf{J}^{(*)}, K_{\mathrm{J}}-1, K_{\mathrm{J}}}\right)^{\mathrm{T}} \in[-1 / 2,1 / 2)^{L_{\mathrm{a}}}
\end{aligned}
$$

whereas the latter one reads as

$$
\mathcal{L}_{2}: \boldsymbol{\alpha}_{\text {ord }} \in[-1 / 2,1 / 2)^{L_{\mathrm{a}}} \longrightarrow \boldsymbol{\alpha}_{\overline{\mathbf{y y}}}{ } \in[-1 / 2,1 / 2)^{L_{\mathrm{a}}} .
$$

Let us form the composite mapping from $[-1 / 2,1 / 2)^{K}$ into $[-1 / 2,1 / 2)^{L_{\mathrm{a}}}$, denoted by $\mathcal{L}_{1} \circ \mathcal{L}_{2}$ : estimation of $\boldsymbol{\nu}_{\text {ord }}$ from the observed vector $\boldsymbol{\alpha}_{\overline{\mathbf{y y}^{*}}}$ is tantamount to inverting the composite mapping $\mathcal{L}_{1} \circ \mathcal{L}_{2}$. Unfortunately, $\mathcal{L}_{1} \circ \mathcal{L}_{2}$ is not invertible unless some a priori knowledge is assumed. Indeed, the linear transformation (56) can be explicitly written as

$$
\boldsymbol{\alpha}_{\mathrm{ord}}=\underbrace{\left(\begin{array}{c}
2 \mathbf{I}_{K} \\
\boldsymbol{\Gamma}
\end{array}\right)}_{\mathbf{B} \in \mathbb{R}^{L_{\mathrm{a}} \times K}} \boldsymbol{\nu}_{\mathrm{ord}}=\mathbf{B} \boldsymbol{\nu}_{\mathrm{ord}}
$$

where the matrix $\Gamma \in \mathbb{R}^{\left(L_{\mathrm{a}}-K\right) \times K}$ - defined in (33) - has exactly two entries in each row equal to 1 , and all other entries equal to 0 . By construction, the matrix $\mathbf{B}$ is full-column rank, i.e., $\operatorname{rank}(\mathbf{B})=K$. On the other hand, the linear mapping (58) is represented by the linear system

$$
\boldsymbol{\alpha}_{\overline{\mathbf{y}} \bar{y}^{*}}=\mathbf{P}_{\overline{\mathbf{y y}}} \boldsymbol{\alpha}_{\text {ord }}
$$

where $\mathbf{P}_{\overline{\mathbf{y y}^{*}}} \in \mathbb{R}^{L_{\mathrm{a}} \times L_{\mathrm{a}}}$ is an unknown permutation matrix, which has been defined in the statement of the Proposition. Since $\mathbf{P}_{\overline{\mathbf{y y}}^{*}}$ is a permutation matrix, it results that $\mathbf{P}_{\overline{\mathbf{y y}}}^{-1}=$ $\mathbf{P}_{\overline{\mathbf{y y}}{ }^{*}}^{\mathrm{T}}$ and, hence, inversion of the composite mapping $\mathcal{L}_{1} \circ \mathcal{L}_{2}$ is equivalent to solving the matrix equation

$$
\mathbf{P}_{\overline{\mathbf{y y}}} \mathbf{B} \nu_{\text {ord }}=\alpha \overline{\mathbf{y y}}^{*}
$$

with respect to the unknowns $\mathbf{P}_{\overline{\mathbf{y y}}^{*}}$ and $\boldsymbol{\nu}_{\text {ord }}$.

\section{APPENDIX B}

\section{PROOF OF PROPOSITION 4.2}

The proof capitalizes on a parameterization of the Toeplitz matrix $\mathbf{T}_{\mathrm{TX}, k, b}$ - defined in (8) with $b \in\{0,1\}$ - in terms of forward shift $\mathbf{F} \in \mathbb{R}^{P \times P}$ and backward shift $\mathbf{B} \triangleq \mathbf{F}^{\mathrm{T}} \in \mathbb{R}^{P \times P}$ matrices [42], whose first column and the first row are given by $[0,1,0, \ldots, 0]^{\mathrm{T}}$ and $[0,1,0, \ldots, 0]$, respectively. One has

$$
\begin{aligned}
& \mathbf{T}_{\mathrm{TX}, k, 0}=\sum_{\ell=0}^{L_{\mathrm{cp}}} \psi\left(\ell T_{\mathrm{c}}-\tau_{\mathrm{TX}, k}\right) \mathbf{F}^{\ell} \\
& \mathbf{T}_{\mathrm{TX}, k, 1}=\sum_{\ell=1}^{L_{\mathrm{cp}}} \psi\left(\ell T_{\mathrm{c}}-\tau_{\mathrm{TX}, k}\right) \mathbf{B}^{P-\ell}
\end{aligned}
$$

where $\tau_{\mathrm{TX}, k} \in\left[0, \Delta_{\max }\right]$ and we remember that the pulse $\psi(t)=\psi_{\mathrm{DAC}}(t) * \psi_{\mathrm{ADC}}(t)$ is known at the CU.

It is crucial to note that

$$
\boldsymbol{\Phi}_{\mathrm{TX}, k} \triangleq \sum_{r=-1}^{1} \boldsymbol{\Phi}_{\mathrm{TX}, k}[r]=g_{\mathrm{TX}, k}^{2} \mathbf{C}_{\mathrm{TX}, k} \boldsymbol{\Omega} \boldsymbol{\Omega}^{\mathrm{T}} \mathbf{C}_{\mathrm{TX}, k}^{\mathrm{T}}
$$

where $\mathbf{C}_{\mathrm{TX}, k} \triangleq \mathbf{T}_{\mathrm{TX}, k, 0}+\mathbf{T}_{\mathrm{TX}, k, 1} \in \mathbb{R}^{P \times P}$ is a circulant matrix [42] by construction, whose first column is given by

$$
\begin{aligned}
\mathbf{c}_{\mathrm{TX}, k} \triangleq\left(\psi\left(-\tau_{\mathrm{TX}, k}\right),\right. & \psi\left(T_{\mathrm{c}}-\tau_{\mathrm{TX}, k}\right), \ldots, \\
& \left.\psi\left(L_{\mathrm{cp}} T_{\mathrm{c}}-\tau_{\mathrm{TX}, k}\right), 0, \ldots, 0\right)^{\mathrm{T}} .
\end{aligned}
$$

Using standard eigenstructure concepts, one gets

$$
\mathbf{C}_{\mathrm{TX}, k}=\mathbf{W}_{P} \operatorname{diag}\left(\mathbf{v}_{\mathrm{TX}, k}\right) \mathbf{W}_{P}^{\mathrm{H}}
$$

where the $p$ th entry of

$$
\mathbf{v}_{\mathrm{TX}, k} \triangleq \sqrt{P} \mathbf{W}_{P}^{\mathrm{H}} \mathbf{c}_{\mathrm{TX}, k} \in \mathbb{C}^{P}
$$

is given by $\left\{\mathbf{v}_{\mathrm{TX}, k}\right\}_{p}=\Psi_{\mathrm{TX}, k}[p] e^{-j \frac{2 \pi}{T} \tau_{\mathrm{TX}, k} p}$, with $\Psi_{\mathrm{TX}, k}[p]$ given by (74)-(75) in Appendix $\mathrm{C}$, for $p \in\{0,1, \ldots, P-1\}$.

In order to acquire the delay $\tau_{\mathrm{TX}, k} \in\left[0, \Delta_{\max }\right]$, for any $k \in\left\{1,2, \ldots, K_{\mathrm{TX}}\right\}$ and $\mathrm{TX} \in\{\mathrm{U}, \mathrm{J}\}$, we observe that, using the eigenstructure of $\mathbf{C}_{\mathrm{TX}, k}$, it results that

$$
\mathbf{W}_{P}^{\mathrm{H}} \boldsymbol{\Phi}_{\mathrm{TX}, k} \mathbf{W}_{P}^{*}=g_{\mathrm{TX}, k}^{2} \mathbf{E}_{\mathrm{TX}, k} \boldsymbol{\Psi}_{\mathrm{TX}, k} \mathbf{\Upsilon} \boldsymbol{\Psi}_{\mathrm{TX}, k} \mathbf{E}_{\mathrm{TX}, k}
$$


where we have defined the matrices

$$
\begin{aligned}
& \mathbf{E}_{\mathrm{TX}, k} \triangleq \operatorname{diag}\left(1, e^{-j \frac{2 \pi}{T} \tau_{\mathrm{TX}, k}}, \ldots, e^{-j \frac{2 \pi}{T} \tau_{\mathrm{TX}, k}(P-1)}\right) \\
& \mathbf{\Psi}_{\mathrm{TX}, k} \triangleq \operatorname{diag}\left(\Psi_{\mathrm{TX}, k}[0], \Psi_{\mathrm{TX}, k}[1], \ldots, \Psi_{\mathrm{TX}, k}[P-1]\right)
\end{aligned}
$$

and $\Upsilon$ has been defined in (48).

For $p \in\{0,1, \ldots, P-1\}$, it is readily seen that the $p$ th diagonal entry of $\mathbf{W}_{P}^{\mathrm{H}} \boldsymbol{\Phi}_{\mathrm{TX}, k} \mathbf{W}_{P}^{*}$ is given by

$$
\left\{\mathbf{W}_{P}^{\mathrm{H}} \boldsymbol{\Phi}_{\mathrm{TX}, k} \mathbf{W}_{P}^{*}\right\}_{p, p}=g_{\mathrm{TX}, k}^{2} \Psi_{\mathrm{TX}, k}^{2}[p]\{\boldsymbol{\Upsilon}\}_{p, p} e^{-j \frac{4 \pi}{T} \tau_{\mathrm{TX}, k} p}
$$

It is shown in Appendix $\mathrm{C}$ that, in the case of zero excess bandwidth, $\Psi_{\mathrm{TX}, k}[0], \Psi_{\mathrm{TX}, k}[1], \ldots, \Psi_{\mathrm{TX}, k}[P / 2-1]$ are equal to the corresponding coefficients of the DFT of $\psi\left(\ell T_{\mathrm{c}}\right)$ and, thus, they are known at the CU. At this point, we are in the position of considering the one-dimensional cost function defined in (46). By resorting to the triangle inequality, one has

$$
\mathcal{I}_{\mathrm{TX}, k}(\beta) \leq\left|g_{\mathrm{TX}, k}\right|^{2} \sum_{p=0}^{P / 2-1}\left|\Psi_{\mathrm{TX}, k}[p]\right|^{4} \mid\left\{\left.\Upsilon_{\}_{p, p}}\right|^{2}\right.
$$

with equality if and only if $\beta=\tau_{\mathrm{TX}, k}+i T / 2(i \in \mathbb{Z})$. This concludes the proof of the statement in Proposition 4.2.

\section{APPENDIX C \\ EXPRESSION OF $\Psi_{\mathrm{TX}, k}[p]$}

Vector $\mathbf{v}_{\mathrm{TX}, k}$ collects the coefficients of the $P$-point DFT of $\mathbf{c}_{\mathrm{TX}, k}$. To evaluate such a DFT, we first observe that the finitelength sequence $\left\{\psi\left(\ell T_{\mathrm{c}}-\tau_{\mathrm{TX}, k}\right)\right\}_{\ell=0}^{L_{\mathrm{cp}}}$ is the sampled version of the waveform $\psi\left(t-\tau_{\mathrm{TX}, k}\right)$ at rate $1 / T_{\mathrm{c}}$. The Fourier transform of $\psi\left(t-\tau_{\mathrm{TX}, k}\right)$ is given by $\Psi(f) e^{-j 2 \pi f \tau_{\mathrm{TX}, k}}$, where $\Psi(f)$ is the Fourier transform of $\psi(t)$ with two-sided bandwidth $1 / T_{\mathrm{c}}$ approximately. ${ }^{9}$ By virtue of the Nyquist-Shannon sampling theorem, the Fourier transform $\bar{\Psi}_{\mathrm{TX}, k}(\nu)$ of the sequence $\psi\left(\ell T_{\mathrm{c}}-\tau_{\mathrm{TX}, k}\right)$ is given by

$$
\bar{\Psi}_{\mathrm{TX}, k}(\nu)=\frac{1}{T_{\mathrm{c}}} \sum_{i=-\infty}^{+\infty} \Psi\left(\frac{\nu-i}{T_{\mathrm{c}}}\right) e^{-j 2 \pi\left(\frac{\nu-i}{T_{\mathrm{c}}}\right) \tau_{\mathrm{TX}, k}} .
$$

The DFT coefficients of $\left\{\psi\left(\ell T_{\mathrm{c}}-\tau_{\mathrm{TX}, k}\right)\right\}_{\ell=0}^{L_{\mathrm{cp}}}$ are samples of $\bar{\Psi}_{\mathrm{TX}, k}(\nu)$ spaced in frequency at integer multiples of $1 / P$. Thus, the $p$ th entry of $\mathbf{v}_{\mathrm{TX}, k}$ reads as

$$
\begin{aligned}
\left\{\mathbf{v}_{\mathrm{TX}, k}\right\}_{p}=\bar{\Psi}_{\mathrm{TX}, k}\left(\frac{p}{P}\right)=\left(e^{-j \frac{2 \pi}{T} \tau_{\mathrm{TX}, k}}\right)^{p} & \cdot \underbrace{\frac{1}{T_{\mathrm{c}}} \sum_{i=-\infty}^{+\infty} \Psi\left(\frac{p-i P}{T}\right) e^{j \frac{2 \pi}{T_{\mathrm{c}}} \chi_{\mathrm{TX}, k} i}}_{\Psi_{\mathrm{TX}, k}[p]}
\end{aligned}
$$

\footnotetext{
${ }^{9}$ We assume zero excess bandwidth. The derivations of this appendix can be straightforwardly generalized to the case of a bandwidth greater than $1 / T_{\mathfrak{c}}$.
}

for $p \in\{0,1, \ldots, P-1\}$. Since $\Psi(f) \approx 0$ for $f \notin$ $\left(-0.5 / T_{\mathrm{c}}, 0.5 / T_{\mathrm{c}}\right)$, it follows that ${ }^{10}$

$$
\begin{aligned}
& \Psi_{\mathrm{TX}, k}[p] \approx \frac{1}{T_{\mathrm{c}}}\left[\Psi\left(\frac{p}{T}\right)+\Psi\left(\frac{p-P}{T}\right) e^{j \frac{2 \pi}{T_{\mathrm{c}}} \chi_{\mathrm{TX}, k}}\right] \\
& =\left\{\begin{array}{c}
\frac{1}{T_{\mathrm{c}}} \Psi\left(\frac{p}{T}\right), \\
\quad \text { for } p \in\left\{0,1, \ldots, \frac{P}{2}-1\right\} \\
\frac{1}{T_{\mathrm{c}}} \Psi\left(\frac{p-P}{T}\right) e^{j \frac{2 \pi}{T_{\mathrm{c}}} \chi_{\mathrm{TX}, k}}, \\
\quad \text { for } p \in\left\{\frac{P}{2}-1, \frac{P}{2}, \ldots, P-1\right\} .
\end{array}\right.
\end{aligned}
$$

If $\tau_{\mathrm{TX}, k}$ is an integer multiple of $T_{\mathrm{c}}$, i.e., the fractional delay $\chi_{\mathrm{TX}, k}$ is zero, then $\Psi_{\mathrm{TX}, k}[p]$ is the $p$ th coefficient of the DFT of the sequence $\psi\left(\ell T_{\mathrm{c}}\right)$. On the other hand, when $\chi_{\mathrm{TX}, k} \neq 0$, the first $P / 2$ coefficients $\Psi_{\mathrm{TX}, k}[0], \Psi_{\mathrm{TX}, k}[1], \ldots, \Psi_{\mathrm{TX}, k}[P / 2-1]$ do not depend on the fractional delay and they are equal to the corresponding coefficients of the DFT of $\psi\left(\ell T_{\mathrm{c}}\right)$, whereas the remaining ones $\Psi_{\mathrm{TX}, k}[P / 2-1], \Psi_{\mathrm{TX}, k}[P / 2], \ldots, \Psi_{\mathrm{TX}, k}[P-1]$ also depend on $\chi_{\mathrm{TX}, k}$.

\section{REFERENCES}

[1] R. Hammons and J. Myers, "Architects of our future: Redefining smart cities to be people-centric and socially responsible," IEEE Internet of Things Magazine, vol. 2, pp. 10-14, June 2019.

[2] Y. Mehmood, F. Ahmad, I. Yaqoob, A. Adnane, M. Imran, and S. Guizani, "Internet-of-Things-based smart cities: Recent advances and challenges," IEEE Commun. Magazine, vol. 55, pp. 16-24, Sept. 2017.

[3] R.M.A. Haseeb-ur-rehman et al., "Sensor cloud frameworks: State-ofthe-art, taxonomy, and research issues," IEEE Sens. J., Early Access.

[4] S.K.u. Zaman et al., "Mobility-aware computational offloading in mobile edge networks: a survey," Cluster Comput., 2021.

[5] R. Jaiswal, A. Agarwal, and R. Negi, "Smart solution for reducing the COVID-19 risk using smart city technology," IET Smart Cities, vol. 2, pp. 82-88, 72020.

[6] P. Kulkarni and T. Farnham, "Smart city wireless connectivity considerations and cost Analysis: Lessons learnt from smart water case studies," IEEE Access, vol. 4, pp. 660-672, 2016.

[7] A. Osorio, M. Calle, J.D. Soto, and J.E. Candelo-Becerra, "Routing in LoRaWAN: Overview and challenges," IEEE Commun. Magazine, vol. 58 , pp. 72-76, June 2020

[8] S. Zhang, H. Zhang, and L. Song, "Beyond D2D: Full dimension UAVto-everything communications in 6G," IEEE Trans. Veh. Technol., vol. 69, pp. 6592-6602, Jun. 2020.

[9] S. Hayat, E. Yanmaz, and R. Muzaffar, "Survey on unmanned aerial vehicle networks for civil applications: A communications viewpoint", IEEE Commun. Surv. Tutor, vol. 18, pp. 2624-2661, 2018.

[10] Q. Wu, W. Mei, and R. Zhang, "Safeguarding wireless network with UAVs: A physical layer security perspective," IEEE Wireless Comm., vol. 26, pp. 12-18, Oct. 2019.

[11] Y. Zou et al., "A survey on wireless security: Technical challenges, recent advances, and future trends," Proc. IEEE, vol. 104, pp. 1727 1765, Sept. 2016

[12] K. Pelechrinis, M. Iliofotou, and S.V. Krishnamurthy, "Denial of service attacks in wireless networks: The Case of jammers," IEEE Commun. Surveys \& Tutorials, vol. 13, pp. 245-57, 2011.

[13] B.B. Zarpelão, R.S. Miani, C.T. Kawakani, and S.C. de Alvarenga, "A survey of intrusion detection in Internet of Things," J. Netw. Comput. Appl., vol. 84, pp. 25-37, 2017.

[14] A. Saeed, A. Ahmadinia, A. Javed, and H. Larijani, "Intelligent intrusion detection in low-power IoTs," ACM Trans. Internet Technol. (TOIT), vol. 16, pp. 1-25, 2016.

[15] M. Wazid, A.K. Das, V. Bhat, and A.V. Vasilakos, "LAM-CIoT: Lightweight authentication mechanism in cloud-based IoT environment," J. Netw. Comput. Appl., vol. 150, pp. 1-16, 2020.

${ }^{10}$ In practice, the integer $P=M+L_{\mathrm{cp}}$ is even since $M$ is a power of 2 and $L_{\mathrm{cp}}=M / 4$. 
[16] M. Wazid, A.K. Das, N. Kumar, A.V. Vasilakos, "Design of secure key management and user authentication scheme for fog computing services," Future Gener. Comput. Syst., vol. 91, pp. 475-492, 2019.

[17] X. Wang et al., "Dynamic spectrum anti-jamming communications: Challenges and opportunities," IEEE Commun. Magazine, vol. 58, pp. 79-85, Feb. 2020.

[18] D. Yang, G. Xue, J. Zhang, A. Richa, and X. Fang, "Coping with a smart jammer in wireless networks: A Stackelberg game approach," IEEE Trans. Wireless Commun., vol. 12, pp. 4038-4047, Aug. 2013.

[19] X. Liu, Y. Xu, L. Jia, Q. Wu, and A. Anpalagan, "Anti-jamming communications using spectrum waterfall: A deep reinforcement learning approach,” IEEE Commun. Lett., vol. 22, pp. 998-1001, May 2018.

[20] P. Zhou and T. Jiang, "Toward optimal adaptive wireless communications in unknown environments," IEEE Trans. Wireless Commun., vol. 15, , pp. 3655-3667, May 2016.

[21] L. Jia, Y. Xu, Y. Sun, S. Feng, L. Yu, and A. Anpalagan, "A multi-domain anti-jamming defense scheme in heterogeneous wireless networks," IEEE Access, vol. 6, pp. 40177-40188, Jun. 2018.

[22] L. Xiao, Y. Chen, W.S. Lin, and K.R. Liu, "Indirect reciprocity security game for large-scale wireless networks," IEEE Trans. Inf. Forensics Secur., vol. 7, pp. 1368-1380, Aug. 2012.

[23] N. Zhang, W. Yu, X. Fu, and S.K. Das, "Maintaining defender's reputation in anomaly detection against insider attacks," IEEE Trans. Syst. Man, Cybern. - Part B: Cybern., vol. 40, pp. 597-611, Jun. 2010.

[24] Y. Xu et al., "A one-leader multi-follower Bayesian-Stackelberg game for anti-jamming transmission in UAV communication networks," IEEE Access, vol. 6, pp. 21697-21709, Apr. 2018.

[25] J. Peng, Z. Zhang, Q. Wu, and B. Zhang, "Anti-jamming communications in UAV swarms: A reinforcement learning approach," IEEE Access, vol. 7, pp. 180532-180543, 2019.

[26] X. Lu, L. Xiao, C. Dai, and H. Dai, "UAV-aided cellular communications with deep reinforcement learning against jamming," IEEE Wireless Commun., vol. 27, pp. 48-53, Aug. 2020.

[27] L. Xiao, C. Xie, M. Min, and W. Zhuang, "User-centric view of unmanned aerial vehicle transmission against smart attacks," IEEE Trans. Veh. Technol., vol. 67, pp. 3420-3430, Apr. 2018.

[28] Y. Wu, W. Fan, W. Yang, X. Sun, and X. Guan, "Robust trajectory and communication design for multi-UAV enabled wireless networks in the presence of jammers," IEEE Access, vol. 8, pp. 2893-2905, Dec. 2019.

[29] M. Mah, H. Lim, and A.W. Tan, "UAV relay flight path planning in the presence of jamming signal," IEEE Access, vol. 7, pp. 40913-40924, Mar. 2019.

[30] B. Duo, Q. Wu, X. Yuan, and R. Zhang, "Anti-jamming 3D trajectory design for UAV-enabled wireless sensor networks under probabilistic LoS channel," IEEE Trans. Veh. Technol., vol. 69, pp. 16288-16293, Dec. 2020.

[31] D. Tse and P. Viswanath, Fundamentals of Wireless Communication Cambridge, U.K.: Cambridge Univ. Press, 2005.

[32] A. Carleial, "Interference channels," IEEE Trans. Inf. Theory, vol. 24, pp. 60-70, Jan. 1978.

[33] B.H. Fleury, M. Tschudin, R. Heddergott, D. Dahlhaus, and K.5Ingeman Pedersen, "Channel parameter estimation in mobile radio environments using the SAGE algorithm," IEEE J. Select. Areas Commun., vol. 17, pp. 434-450, Mar. 1999.

[34] W. A. Gardner, Introduction to Random Processes. New York: Mc-GrawHill, 1990.

[35] Y. Li, P. Hui, D. Jin, and S. Chen, "Delay-tolerant network protocol testing and evaluation," IEEE Commun. Magazine, vol. 53, pp. 258-266, Jan. 2015.

[36] S. Serbetli and A. Yener, "Transceiver optimization for multiuser MIMO systems," IEEE Trans. Signal Process., vol. 52, pp. 214-226, Jan. 2004.

[37] W.A. Gardner and C. Chen, "Signal-selective time-difference-of-arrival estimation for passive location of man-made signal sources in highly corruptive environments - Part I: Theory and method," IEEE Trans. Signal Process., vol. 40, pp. 1168-1184, May 1992.

[38] C. Chen and W.A. Gardner, "Signal-selective time-difference of arrival estimation for passive location of man-made signal sources in highly corruptive environments - Part II: Algorithms and performance," IEEE Trans. Signal Process., vol. 40, pp. 1185-1197, May 1992.

[39] G. Gelli, "Power and timing parameter estimation of multiple cyclostationary signals from sensor array data," Signal Process., vol. 42, pp. 97-102, 1995.

[40] P. Ciblat, P. Loubaton, E. Serpedin, and G.B. Giannakis, "Performance analysis of blind carrier frequency offset estimators for noncircular transmissions through frequency-selective channels," IEEE Trans. Signal Process., vol. 50, pp. 130-140, Jan. 2002.

[41] A. Napolitano, Cyclostationary Processes and Time Series: Theory, Applications, and Generalizations. Academic Press, 2019.

[42] R.A. Horn and C.R. Johnson, Matrix Analysis. New York: Cambridge Univ. Press, 1990.

[43] J. Tang, M. Dabaghchian, K. Zeng, and H. Wen, "Impact of mobility on physical layer security over wireless fading channels," IEEE Trans. Wireless Commun., vol. 17, pp. 7849-7864, Dec. 2018.

[44] H.B. Mann, Addition Theorems: The Addition Theorems of Group Theory and Number Theory. Wiley, 1965.

[45] P.A. Bello, "Characterization of randomly time-variant linear channels," IEEE Trans. Commun. Syst., vol. 11, pp. 360-393, Nov. 1963.

[46] J.G. Proakis, Digital Communications. New York, NY, USA: McGrawHill, 2001.

[47] G. Matz, H. Bolcskei, and F. Hlawatsch, "Time-frequency foundations of communications: Concepts and tools," in IEEE Signal Process. Mag. vol. 30, pp. 87-96, Nov. 2013.

[48] A. Napolitano, Generalizations of cyclostationary signal processing: Spectral analysis and applications. Hoboken, NJ, USA: Wiley 2012.

[49] M. Wax and T. Kailath, "Detection of signals by information theoretic criteria," IEEE Trans. Acoust. Speech, Signal Processing, vol. ASSP-33, pp. 387-392, Apr. 1985.

[50] T.M. Cover and J.A. Thomas, Elements of Information Theory. New York: Wiley, 1991.

[51] A.D. Wyner, "Recent results in the Shannon theory," IEEE Trans. Inf. Theory, vol. 20, pp. 2-10, Jan. 1974.

[52] D.N. Tse and S.V. Hanly, "Multiaccess fading channels. I. Polymatroid structure, optimal resource allocation and throughput capacities," IEEE Trans. Inf. Theory, vol. 44, pp. 2796-2815, Nov. 1998.

[53] B. Picinbono and P. Chevalier, "Widely linear estimation with complex data," IEEE Trans. Signal Process., vol. 43, pp. 2030-2033, Aug. 1995.

[54] P. Li, D. Paul, R. Narasimhan, and J. Cioffi, "On the distribution of SINR for the MMSE MIMO receiver and performance analysis," IEEE Trans. Inf. Theory, vol. 52, pp. 271-286, Jan. 2006.

[55] A. Björck, Numerical Methods for Least Squares Problems. SIAM: Philadelphia, PA, 1996.

[56] L. Franks, "Polyperiodic linear filtering," in Cyclostationarity in Communications and Signal Processing, W. A. Gardner, Ed., pp. 240-266, IEEE Press, Piscataway, NJ, USA, 1994.

[57] C. Corduneanu, Almost Periodic Functions. Chelsea Publishing Company New York, 1989.

[58] S.M. Kay, Fundamentals of Statistical Signal Processing: Estimation Theory. Englewood Cliffs, NJ: Prentice-Hall, 1993.

[59] Y. Zeng, R. Zhang, and T.J. Lim, "Wireless communications with unmanned aerial vehicles: Opportunities and challenges," IEEE Commun. Mag., vol. 54, pp. 36-42, May 2016.

[60] A.V. Dandawaté and G.B. Giannakis, "Asymptotic theory of mixed time averages and $k$ th-order cyclic moment and cumulant statistics," IEEE Trans. Inf. Theory, vol. 41, pp. 216-232, Jan. 1995.

[61] W.A. Gardner and C.M. Spooner, "The cumulant theory of cyclostationary time-series - Part I: Foundation," IEEE Trans. Signal Process., vol. 42, pp. 3387-3408, Dec. 1994.

[62] E. Moulines, P. Duhamel, J.-F. Cardoso, and S. Mayrargue, "Subspace methods for the blind identification of multichannel FIR filters," IEEE Trans. Signal Process., vol. 43, pp. 516-525, Feb. 1995.

[63] D. Darsena, G. Gelli, L. Paura, and F. Verde, "Widely linear equalization and blind channel identification for interference-contaminated multicarrier systems," IEEE Trans. Signal Process., vol. 53, pp. 1163-1177, Mar. 2005.

[64] E. Haas, "Aeronautical channel modeling," IEEE Trans. Veh. Technol., vol. 51, pp. 254-264, Mar. 2002. 